\title{
The multi-depot open location routing problem with a heterogeneous fixed fleet
}

\author{
Samuel Nucamendi-Guillén ${ }^{\mathrm{a}}$, Alejandra Gómez Padilla ${ }^{\mathrm{b}}$, Elias Olivares-Benitez ${ }^{\mathrm{a}, *}$, \\ J. Marcos Moreno-Vega ${ }^{c}$ \\ ${ }^{a}$ Facultad de Ingeniería, Universidad Panamericana. Álvaro del Portillo 49, Zapopan, Jalisco, 45010, Mexico

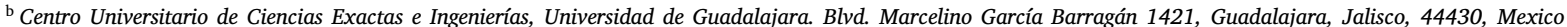 \\ ${ }^{\mathrm{c}}$ Dpto. de Ingeniería Informática y de Sistemas, Universidad de La Laguna. Avenida Astrofísico Francisco Sánchez s/n. San Cristóbal de La Laguna, Santa Cruz de \\ Tenerife, 38200, Spain
}

\section{A R T I C L E I N F O}

\section{Keywords:}

Multi-depot vehicle routing problem

Open vehicle routing problem

Location-routing problem

Heterogeneous fixed fleet

Metaheuristic

Third-party logistics

\begin{abstract}
A B S T R A C T
This paper introduces the multi-depot open location routing problem (MD-OLRP) with a heterogeneous fixed fleet. The problem is inspired by the collection problem of a company which collects raw materials from different suppliers coordinating several carriers. Each carrier has a heterogeneous fixed fleet. Moreover, there is a fixed cost for contracting each vehicle and a variable cost associated with the distance traveled. The empty haul return to the vehicles depot is not considered in the cost. The raw materials collected are delivered to a single delivery point. The problem is modeled as a Mixed Integer Linear Program (MILP) that minimizes the total cost, selecting the carriers to be contracted, the vehicles to be used from each contracted carrier and the collection routes. For small instances, the model can be solved to optimality. However, approximate procedures are necessary to handle larger instances. In this sense, in the present work we propose an intelligent metaheuristic which incorporates problem specific knowledge to solve it. The computational results show that the solution method is computationally efficient and provides high quality solutions. In particular, the new solution obtained for the case of study generates savings of $30.86 \%$ to the company.

The main contributions of the paper are the new problem statement that was not found in the literature, its association to the real problem of a company and the intelligent metaheuristic proposed to solve it. Additional experimentation used the model proposed to solve a simpler problem obtaining new best solutions compared to those reported in the recent literature.
\end{abstract}

\section{Introduction}

This study is motivated by the problem of a company located in Mexico. This company orders raw material from several suppliers located at the United States of America (USA), and transports this raw material by one carrier company. Every week, the company informs the carrier the number of units to be picked and the picking addresses, and the carrier must collect the units to deliver them at border customs.

When analyzing the different costs incurred per year, the finance department realized that the transportation costs were excessive. It was then decided to analyze if it would be possible to reduce transportation costs by contracting the service from several carriers. Under these premises, the company's logistics planner was able to provide a lower cost solution for a small-sized instance. However, this solution was improved by using a solution method which incorporates problem specific knowledge (intelligence).
In this work, we consider several carriers, each with different fleet composition and established at different cities, in order to decide which supplier(s) will be served by which carrier(s) and which type of vehicle will be used. Even when this problem belongs to the class of vehicle routing problems, given its particular characteristics, it differs from the classic routing approaches addressed, including those that consider heterogeneous fleet.

In particular, the problem faced by this company differs from the typical vehicle routing approaches in the fact that it considers several possible origins (carriers), each with a particular heterogeneous fleet of vehicles (different capacity and contracting costs). The contracted vehicles must collect a number of units (raw material) from several locations (suppliers) and deliver them at a single point (border customs) located at the frontier between Mexico and USA. The fleet composition

\footnotetext{
* Corresponding author.

E-mail addresses: snucamendi@up.edu.mx (S. Nucamendi-Guillén), alejandra.gomez@cucei.udg.mx (A. Gómez Padilla), eolivaresb@up.edu.mx
} (E. Olivares-Benitez), jmmoreno@ull.es (J.M. Moreno-Vega). 
and the routes have to be decided under total cost minimization criteria. The empty haul back to the depot is not considered in routing design.

From the theoretical perspective, this class of problems has gained attraction among researchers because of its interesting mathematical complexity. To the best of the authors' knowledge, a similar problem has been widely studied in the literature that considers homogeneous capacities and a specific destination for each route (the multi-depot open vehicle routing problem, MDOVRP). However, no research has tailored to the particular situation in which multiple depots, open routes, heterogeneous fleet, common destination, and contracting costs are considered. Thus, the contribution of this paper to state of the art in the literature lies in the fact of proposing new mathematical models and algorithms to deal with more complex situations and that can solve the classic MDOVRP.

For the problem under study, a new mixed integer formulation is proposed in which routes and the fleet composition are decided simultaneously to ensure that all the raw material is collected. For small instances, the model can be solved to optimality. However, approximate procedures are necessary to handle larger instances. In this sense, in the present work we propose a solution method based on a multistart metaheuristic.

Heuristics and metaheuristics are solution methods that develop an intelligent exploration of the search space to find high quality solutions. As Verdegay et al. (2008) established, heuristics and metaheuristics constitute, together with probabilistic models, fuzzy and multivalued logics and neural networks, the basic components of soft computing. These tools are used to build intelligent systems in different domains. Some efficient implementations can be found in Moura and Oliveira (2005), Yu et al. (2020) and Zhao et al. (2020).

Problem specific knowledge can help to increase the efficiency and effectiveness of a metaheuristic. Our solution method consists of a constructive phase and an improvement phase that are executed iteratively until the stop criterion is met. In the constructive phase, a feasible solution is obtained by randomly selecting a subset of vehicles with sufficient total capacity to collect the raw material from the suppliers, prioritizing the trade-off between capacity and contracting cost. Then the suppliers are clustered and served by one of these vehicles. In the improvement phase, the obtained solution is improved by applying a mechanism based on local search. We use problem specific knowledge to design the previous phases.

Our proposal can be used as part of a decision support system to identify high quality schedules. The system should also incorporate a model to estimate the demand for raw material. Using the estimated demand as input, our solution method provides a minimum cost quality planning. We consider the design and implementation of such a system as a future line of work.

A metaheuristic procedure that solves the problem in reasonable computation time is also presented. Even when the algorithm was appropriately designed to deal with the case study, its robustness was also verified by solving theoretical scenarios based on adapting benchmark instances proposed in the literature for similar problems. Both the model and the algorithm were also implemented using instances for the classic MDOVRP and their results were compared with the formulations and relevant methods reported in the literature.

\section{Literature review}

According to Christofides and Eilon (1969), the vehicle routing problem (VRP) was introduced by Dantzig and Ramser (1959) under the name of the "truck dispatching problem". The problem was initially defined as "A set of customers, each with a known location and a known requirement for some commodity, is to be supplied from a single depot by delivery vehicles of known capacity". In addition, Christofides and Eilon (1969) proposed three solution methods: branch and bound, savings, and 3-optimal tour. Ten problems (instances) were considered and found that the 3-optimal tour produced the best results. Five years later, Gillett and Miller (1974) proposed a sweep algorithm to solve the VRP using polar-coordinate angles of locations.

The problem proposed in our research has four particular characteristics in the domain of vehicle routing problems. The first one is the multiple depot feature. Differently to most of the vehicle routing problems where only one depot is considered, in our case multiple depots are considered. This characteristic receives an expanded complexity because these depots are not considered as effective operational, but a location decision will result in using only some of them. The second feature is then the decision that has to be made regarding which depots (carriers) will be the starting point of a route. The third distinguishing feature of our problem is the use of a heterogeneous fleet. In the earliest versions of the vehicle routing problem only homogeneous fleet was considered, i.e. all vehicles have the same characteristics. In our case, each depot has a fleet composed of different types and number of vehicles, and these fleets may be different between depots. A fourth feature of the problem is that routing tours are "open", i.e. the tour does not consider the return to the depot. In practice, transportation companies include the empty-haul return into the costs, but this feature opens the door to select distant depots when empty-haul costs are negligible.

Finally, unlike the traditional location routing problems, variable costs are associated for each type of contracted vehicle (instead of having a fixed opening cost for the selected depots). These costs are independent for each origin. In addition, since each potential origin has a number of vehicles available, it could be (eventually) possible that all of the required vehicles depart from the same origin.

The following lines in this section will describe first the earliest works on these characteristics along with the most recent examples. Later, those works that combine some of these characteristics will be described in order to differentiate our scientific contribution beyond the fact that our study was motivated by a practical need from a company.

In the multi-depot multiple vehicle routing problems (MDVRP), each vehicle must leave and return to the same depot as with the VRP, but customers are serviced from one of several depots (Kim et al., 2011). The first description and treatment of the multiple depot vehicle routing problem is due to Wren and Holliday (1972). They used a two-step heuristic where an initial solution is constructed and later is refined.

Subsequently, Gillett and Johnson (1976) also addressed this problem using a modification of the sweep algorithm to solve eleven instances with 2 to 4 depots and 50-249 customers. The MDVRP is well-known as a combinatorial optimization problem, and it is shown to be NP-complete, since it can be reduced to the classical VRP which is known to be NP-hard (Salhi et al., 2014; Tang et al., 2010). Tang et al. (2010) proposed a solution with a multi-objective genetic algorithm. With an application to reverse logistics, Kim et al. (2011) proposed a multi-depot vehicle-routing method to minimize distance traveled for MDVRP. They designed a two-stage solution procedure: first, they determine which client will be served from which depot and, second, they generated routes for each vehicle-based at a depot. Zhu et al. (2015) proposed a solution with particle swarm optimization and their problem considered 2-dimensional weighted items. Juan et al. (2015) proposed a hybrid metaheuristic combining iterated local search and biased randomization to solve the MDVRP. Wang and Lin (2017) decomposed the MDVRP into several VRPs which are then decomposed into traveling salesman problem (TSP). They proposed a hybrid mosquito host-seeking algorithm optimized with the 3-opt local method. Bezerra et al. (2018) addressed a MDVRP using a General Variable Neighborhood Search with a random neighborhood ordering scheme. They solved a set of benchmark instances available with results of up to $4.41 \%$ over the best known solution. A complete literature review of MDVRP may be found in Eksioglu et al. (2009), Montoya-Torres et al. (2015), and Braekers et al. (2016). 
Recently, Lalla-Ruiz and Voß (2019) studied a customer-centric variant of the MDVRP, named as the multi-depot cumulative capacitated vehicle routing problem (MDCCVRP) and addressed it via two approaches: (1) a mathematical formulation enhanced by incorporating lower bound inequalities, and (2) an optimization metaheuristic under special intensification conditions. However, their problem differs form our approach because they considered a different objective function, and they set the number of depots and fixed the number of vehicles with homogeneous capacity.

Regarding the variant of the MDVRP that considers open routes (MDVORP) problem was introduced in the literature by Tarantilis and Kiranoudis (2002) to tackle a distribution problem of fresh meat, considering a homogeneous fleet of vehicles. Since then, relevant studies such as (Lalla-Ruiz et al., 2016; Lalla-Ruiz \& Mes, 2020; Liu et al., 2014; Soto et al., 2017) proposed mathematical formulations and efficient metaheuristic algorithms to handle this problem.

The Heterogeneous Fleet Vehicle Routing Problem (HFVRP) is a variant of the VRP with vehicles of different capacities and costs (Penna et al., 2013). There are two basic versions of this problem. In one version, the composition of the fleet is fixed, and the number of vehicles per type is known, proposed by Taillard (1999), and known also as Heterogeneous Fixed Fleet Vehicle Routing Problem (HFFVRP). Another version of the problem considers an infinite availability of vehicles of different capacities, proposed by Golden et al. (1984), and also named as the Fleet Size and Mix Vehicle Routing Problem (FSMVRP). In the first case, the number of vehicles is a constraint but in the presence of a large number of vehicles, not all of them may be used. In the second version, part of the decision is to determine the composition of the fleet. The case presented in our research is better associated to the Heterogeneous Fixed Fleet Vehicle Routing Problem, since the size of the fleet is known for each of the depots but in our case, we determine the composition of the fleet as in the FSMVRP.

Euchi and Chabchoub (2010) proposed a metaheuristic solution based on tabu search to solve the HFFVRP and tested it for up to 15 vehicles, verifying the performance with respect to the quality of the solution and computation time. Markov et al. (2015) solved a waste collection problem integrating a heterogeneous fixed fleet and flexible assignment of origin and destination depot. They propose a local search heuristic that first proposes an initial feasible solution and then improves these solutions by an iterative procedure that considers infeasible intermediate solutions. Wang et al. (2014) addressed the HFFVRP with multi-compartment vehicles. This variation allows transporting different types of products with special requirements into the vehicles. This additional level of availability in the resources and demand increases the complexity with respect to the original problem. They designed a reactive guided tabu search (RGTS) to solve 8 instances and compared different guiding mechanisms.

The Open Vehicle Routing Problem (OVRP) is a variant of the VRP in which vehicles do not need to return to the depot. This problem was introduced by Schrage (1981), and later Sariklis and Powell (2000) named it formally as OVRP (Soto et al., 2017). Niu et al. (2018) studied an OVRP with fuel consumption constraint to reduce the environmental impact. They developed a tabu search algorithm to solve instances of up to 120 nodes based on Beijing roads.

Given the nature of Vehicle Routing Problems of having a high number of variants, also the combination of these variants produce a great number of situations. The study of these combinations results in a wide body of literature addressing complex problems that require sophisticated methods to solve them. In the following paragraphs we will explain some of these combinations found in literature related to the characteristics of our problem.

Soto et al. (2017) studied a multi-depot open vehicle routing problem (MDOVRP), which combines the MDVRP and the OVRP. They proposed a solution method based on a hybridization of Multiple Neighborhood Search with Tabu Search and Ejection Chains. They solved sets of instances modified from benchmark capacitated VRP instances.
An interesting logistic problem for cargo aircraft was solved by Aksoy and Kapanoglu (2012) as a multi-commodity, multi-depot and heterogeneous vehicle pickup and delivery problem with a mixed integer linear program (MILP). In this problem, the vehicles load from any of the pickup points and deliver to each delivery point a variety of supplies. Lahyani et al. (2018) modeled the Multi-depot Fleet Size and Mix VRP with five different formulations, identifying which ones provide better results. The Fleet Size and Mix component is similar to the Heterogeneous Fixed Fleet variant, but with unlimited number of vehicles. In both cases these problems do not contain the Open and Location components.

Regarding the Location Routing Problem (LRP), most of the authors study this problem from the point of view of minimizing distribution cost. In other words, the manufacturer must decide for the best strategy for delivery. An interesting and extensive review related to this perspective can be found in Drexl and Schneider (2015). In particular, Markov et al. (2015) studied a waste collection problem that is a vehicle routing problem with heterogeneous fixed fleet and a more general version of the open location routing problem. In their case, the assignment of origin and destination depot is flexible. They proposed a mixed integer linear program enhanced with valid inequalities, but for solving large instances they implemented a local search heuristic.

Within the literature reviewed only three works were found which considered the MDOLRP. First, Yu and Lin (2015) introduced the combination of the location decision and the open routing structure. Their research was motivated from the rise in contracting with thirdparty logistic and consider the open-routing approach, but consider fixed costs at the moment of contracting the vehicles (they used a homogeneous fleet). As a mechanism of solution, they developed a simulated annealing heuristic implemented over a set of adapted instances from Ting and Chen (2013) (up to 318 customer nodes and 15 origin nodes). Secondly, Koç et al. (2016) addressed the locationrouting problem from the perspective of emissions and analyzed the effect of parameters such as depot cost/location, fuel consumption and operational costs. Although these works share features with our problem, they consider a homogeneous fleet while we are modeling a heterogeneous fleet. Third, Pichka et al. (2018) studied a two echelon open location routing problem and developed mixed-integer linear programming models and a hybrid heuristic approach (based on simulated annealing). The heuristic divides the problem by echelon and the simulated annealing is used in both sub-problems. The effectiveness of their approach was validated using instances obtained from the literature.

To the best of our knowledge, none of the described formulations and algorithms were particularly suitable to solve the problem under study. We decided to adapt the formulations and procedures described in Angel-Bello et al. (2017) and Nucamendi-Guillén et al. (2018) to develop an appropriate mathematical formulation and a metaheuristic procedure to address the MD-OLRP. Angel-Bello et al. (2017) propose mathematical models, some derived from the multiple traveling salesman problem, and proposed time-dependent formulations to reduce the number of binary variables required. Nucamendi-Guillén et al. (2018) propose two metaheuristic procedures to solve the Cumulative Capacitated Vehicle Routing Problem (CCVRP).

In summary, the problem studied here has the following characteristics: (1) A set of depots (which are our carriers) exist, each with heterogeneous fleet of vehicles; (2) A route may start at any origin but all of them end at the same delivery point; (3) Customers (which are in our case the suppliers of raw material) must be visited once by a vehicle (demand cannot be split); (4) The capacity of each vehicle must not be exceeded and, (5) A trade-off between routing and contracting costs is sought. 


\section{Problem description}

In this work, a collecting problem for a manufacturing plant is addressed. The company receives the raw materials in Nuevo Laredo (border customs) from different suppliers located in USA. The collection is done via third-party companies. Based on the agreement with the suppliers, the company under study manages this service.

Formally, the problem can be formulated as follows: Let $O$ be the set of carriers, $P=\{1,2, \ldots, n+1\}$ be the set of nodes to visit, were $P^{\prime}=\{1,2, \ldots, n\}$ denotes the suppliers set and node $n+1$ corresponds to the location of the delivery point. Also, let $F$ be the set of potential carriers to contract. Each carrier has a heterogeneous limited fleet of vehicles, each vehicle $r$ with a particular contracting cost and capacity. Associated with each supplier there is an amount of raw material that must be collected and transported to the delivery point. Routes must be designed to minimize the costs of contracting (fixed fee) and transporting (based on distance) without exceeding the capacity of the vehicles. Therefore, this is a problem of designing routes with different potential origins (carriers) and a single destination (delivery point).

The following assumptions are made:

1. Service times are not considered for the problem under study

2. The cost of empty-haul return is absorbed by the carriers,

3. The demands (of raw material) are deterministic and quantified in pallets, and

4. The traveled distance of the routes is properly translated into monetary costs to standardize the objective function.

A solution $(S)$, for this problem is composed of a set of disjoint routes that, departing from their corresponding origins (carriers), all of them end in the delivery point. Each supplier is visited by only one vehicle, which collects completely the demand without exceeding the capacity of the vehicle.

\section{Mathematical formulation}

This section provides the mathematical formulation for the problem under study.

Let:

$$
\begin{aligned}
& n=\text { number of suppliers to attend } \\
& m=\text { number of available origins (carriers) to } \\
& \text { consider for contracting vehicles } \\
& P^{\prime}=\left\{p_{1}, p_{2}, \ldots, p_{n}\right\}, \quad \text { set of suppliers } \\
& d_{j} \text {, demand of the } j \text { th supplier } \\
& Q=\sum_{j=1}^{n} d_{j}, \quad \text { total demand of raw material } \\
& F=\left\{f_{1}, f_{2}, \ldots, f_{m}\right\}, \quad \text { set of carriers } \\
& R_{i} \text {, set of vehicles for carrier } f_{i} \\
& i=1,2, \ldots, m \\
& k_{i} \text {, number of available vehicles for carrier } f_{i} \\
& i=1,2, \ldots, m \\
& Q_{i}^{r} \text {, Capacity of the } r \text { th vehicle for carrier } f_{i} \\
& i=1,2, \ldots, m ; r=1,2, \ldots, k_{i} \\
& Q_{\max } \text {, maximum capacity of any vehicle } \\
& w_{i}^{r} \text {, contracting (fixed) cost for the } r \text { th vehicle } \\
& \text { of carrier } f_{i} \\
& i=1,2, \ldots, m ; r=1,2, \ldots, k_{i}
\end{aligned}
$$

$$
\begin{aligned}
R=\bigcup_{i=1}^{m} R_{i} & \text { Set of total available vehicles } \\
D_{i j} & \text { transportation cost between nodes (carriers) } i \\
& \text { and suppliers } j \\
C_{i j} \quad & \text { transportation cost between nodes (suppliers) } \\
& i \text { and } j
\end{aligned}
$$

When needed, to simplify notation, $f(r), q(r)$ and $w(r)$ will be used to denote the carrier (origin), capacity and contracting cost of vehicle $r$. Likewise, $c_{i j}$ will be used instead of $C(i, j)$ when required. The transportation cost for each arc is calculated as the distance multiplied by the corresponding rate $(\$ / \mathrm{km})$.

The variables associated to the model are the following:

Let $o_{i j}^{r}$ be equal to 1 if the arc $(i, j)$ is used for transportation between carrier $i$ and the first node $j$ through vehicle $r$ and equal to zero otherwise. Let $x_{i j}$ be equal to 1 if the $\operatorname{arc}(i, j)$ is used for transportation between nodes $i$ and $j$ and equal to zero otherwise. Let $z_{i}^{r}$ be equal to 1 if vehicle $r$ is contracted from carrier $i$ and equal to zero otherwise. Let $v_{i j}^{r}$ denotes the sum of the remaining demands of the route after departing from carrier $i$ using vehicle $r$ when $o_{i j}^{r}=1$. In the same way, $r_{i j}$ indicates the sum of remaining demands after visiting supplier $i$ when $x_{i j}=1$.

Our formulation is stated as follows:

Minimize:

$\sum_{i=1}^{m} \sum_{j=1}^{n} D_{i j} \sum_{r=1}^{\left|R_{i}\right|} o_{i j}^{r}+\sum_{i=1}^{n} \sum_{\substack{j=1 \\ j \neq i}}^{n+1} C_{i j} x_{i j}+\sum_{i=1}^{m} \sum_{r=1}^{\left|R_{i}\right|} w_{i}^{r} z_{i}^{r}$,

subject to:

$\sum_{j=1}^{n} o_{i j}^{r} \leq 1$ $\forall i \in F ; r \in R_{i}$,

$\sum_{i=1}^{m} \sum_{r=1}^{\left|R_{i}\right|} o_{i j}^{r}+\sum_{\substack{i=1 \\ i \neq j}}^{n} x_{i j}=1$,

$\forall j \in P^{\prime}$,

$\sum_{\substack{i=1 \\ i \neq j}}^{n+1} x_{j i}=1$

$\forall j \in P^{\prime}$,

$z_{i}^{r} \geq \sum_{j=1}^{n} o_{i j}^{r}$,

$\forall i \in F ; r \in R_{i}$,

$v_{i j}^{r} \geq d_{j} o_{i j}^{r}$,

$\forall i \in F ; j \in P^{\prime} ; r \in R_{i}$,

$v_{i j}^{r} \leq Q_{i}^{r} o_{i j}^{r}$,

$\forall i \in F ; j \in P^{\prime} ; r \in R_{i}$,

$r_{i j} \geq d_{j} x_{i j}$,

$\forall i \in P^{\prime} ; j \in P^{\prime} ; i \neq j$

$r_{i j} \leq\left(Q_{\max }-d_{i}\right) x_{i j}$,

$\forall i \in P^{\prime} ; j \in P ; i \neq j$

$\sum_{i=1}^{m} \sum_{r=1}^{\left|R_{i}\right|} v_{i k}^{r}+\sum_{\substack{i=1 \\ i \neq k}}^{n} r_{i k}-\sum_{\substack{j=1 \\ j \neq k}}^{n+1} r_{k j}=d_{k}$,

$\forall k \in P^{\prime}$

$o_{i j}^{r} \in\{0,1\}$,

$\forall i \in F ; j \in P^{\prime} ; r \in R_{i}$,

$x_{i j} \in\{0,1\}$,

$z_{i}^{r} \in\{0,1\}$,

$v_{i j}^{r} \geq 0$,

$\forall i \in F ; j \in P^{\prime} ; r \in R_{i}$,

$r_{i j} \geq 0$,

$\forall i \in P^{\prime} ; j \in P$.

In this formulation, the objective function (1) aims at minimizing the total cost incurred by transportation and contracting. Constraints (2) allow that only active carriers must be considered for constructing each route. Constraints (3) and (4) force suppliers to be visited only once. It can be noticed that constraints (2) and (3) ensure that only one vehicle is assigned to start the route and, in particular, constraints (4) 


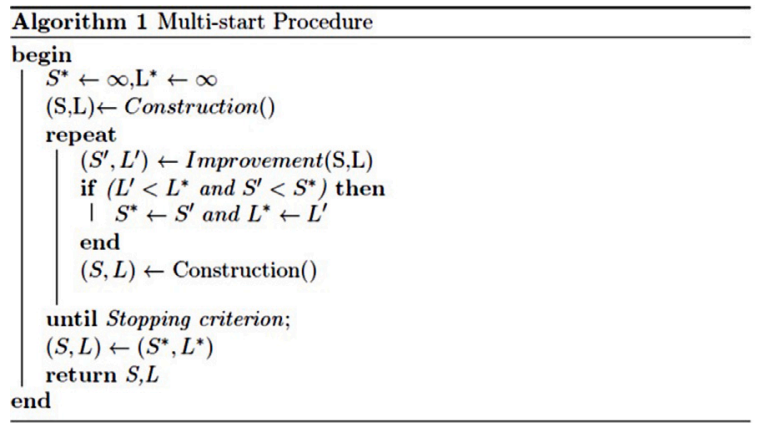

Fig. 1. Outline for the multi-start procedure.

ensure that all of the vehicles end their routes at the final destination $(n+1)$. Constraints (5) force to use vehicles only from active carriers. Constraints (6) and (8) establish the minimum values for $v_{i j}^{k}$ and $r_{i j}$, and allows to compute the minimum capacity required to collect the demand of supplier $j$. Constraints (7) and (9) force the same variables to be zero when $o_{i j}^{r}$ and $x_{i j}$ are zero. For these constraints, $Q_{i}^{r}$ ensures that the route assigned to vehicle $r$ departing from carrier $i$ does not exceed its capacity while $Q_{\max }$ establishes an upper bound for the sum of the demands of the remaining nodes in any active route. Constraints (10) allow to meet the demand with an active vehicle and avoid sub-tours. Finally, constraints (11) to (15) establish the domain of the variables.

\section{Metaheuristic algorithm}

This section describes the metaheuristic procedure developed for solving the Multi-depot Open Location Routing Problem with Heterogeneous Fixed Fleet. It consists of a multi-start algorithm with two phases: construction and improvement. During the constructive phase, a subset of vehicles is selected and the construction of routes is done (initial solution). The cost any constructed solution is denoted as $L$. The initial solution is then improved by applying different local searches in the improvement phase. Fig. 1 exhibits the pseudocode of the metaheuristic procedure.

It is important to mention here that we explored an Iterated Local Search (ILS) approach during the process of the design of the algorithm. However, due to the capacity constraints, the perturbation was not as successful as the reconstruction process, and also required longer computational times to obtain a new feasible initial solution. Due to this, we decided to implement a partial destroy-construction procedure, which reduced the probability of infeasibility, but being aware that the construction process could lead to a previous initial solution, i.e. a solution previously explored.

Next, detailed descriptions of the constructive (Section 5.1) and improvement (Section 5.2) phases are provided.

\subsection{Constructive phase}

A feasible solution for this problem is composed by a subset of vehicles capable of collecting the raw material from suppliers. It should be observed that it is not enough that the sum of the capacities of the vehicles is greater than the sum of all demands, because the capacity of each vehicle must not be exceeded (and for each supplier, its demand must be collected entirely by only one vehicle).

Our constructive method selects, on a first place, a subset of vehicles with enough total capacity. Then, each supplier is assigned to one vehicle by procuring feasibility in terms of capacity. We incorporate problem specific knowledge to design these components of the solution method. Thus, the vehicles are selected using a probability distribution which depends on their capacity, distance to the delivery point and contracting cost. The assignment is carried out by clustering the suppliers according to the proximity criterion.

\subsubsection{Computation of the number of vehicles to use}

The number of required vehicles to serve the total demand depends on both the capacity of the vehicles and the demand of each supplier. For instance, if two orders should be collected from two different suppliers with respective values of 10 and 15 units of raw material, and the carrier owns two vehicles with 20 and 15 units of capacity, the required amount of vehicles is 2 . By the contrary, if the size of the orders are 10 and 15 units, and the capacities of the vehicles are 20 are 30 units, it would be enough to use only one vehicle to collect the total demand (the one with capacity of 30 units).

To determine the number of vehicles, we apply a heuristic procedure that takes into account the demand of raw material, the distances between carriers and the delivery point, the contracting costs and the capacities of the vehicles, as shown in Fig. 2. The procedure iteratively selects vehicles until the total demand of raw material is fulfilled. The algorithm tends to select vehicles with high capacities, low contracting costs, and close to the manufacturing company.

Let $Q$ be the total demand of raw material, $F=\left\{f_{1}, f_{2}, \ldots, f_{m}\right\}$ be the set of carriers and $k_{i}$ be the number of vehicles of carrier $i$ $(i=1,2, \ldots, m)$. Let $q_{i}^{r}$ y $w_{i}^{r}$, be the capacity and the contracting cost of the $r$ th vehicle from carrier $i$, respectively. Also, let $c_{i, n+1}$ be the distance between carrier $i$ and the delivery point (customs location) $n+1$. Associated to each vehicle $r$ from carrier $i$, we compute the index $I_{i}^{r}=\frac{Q_{i}^{r}}{\left(c_{i, n+1}+w_{i}^{r}\right)}$.

The procedure iteratively chooses the vehicles based on the previous index using a roulette wheel selection. Therefore, the probability of selection for vehicle $r$ from carrier (freighter) $i$ is estimated as:

$p_{i}^{r}=\frac{I_{i}^{r}}{\sum_{i} \sum_{r} I_{i}^{r}}$.

Every single time a vehicle is selected, it is removed from the list of available vehicles and its capacity is subtracted from the total demand to collect. Additionally, the probabilities of selection are updated for the remaining vehicles. The procedure ends when the sum of the capacities for the selected vehicles is greater than or equal to the total demand to collect. Let $R^{\prime} \subseteq R$ be the subset of selected vehicles.

It is important to note that the subset of vehicles selected by the previous procedure does not guarantee feasibility for the initial solution. For instance, when on any moment during the construction of the routes, the demand of one supplier equals to the sum of the remaining capacities of the selected vehicles, but it is greater that any of them separately. This is why our heuristic artificially increases the total demand to collect (by using a parameter), and the selection of vehicles ends when the total artificial demand is satisfied (or exceeded). More specifically, given the total demand $Q$, we compute the total artificial demand as $Q^{\prime}=(1+\delta \cdot Q)$. The procedure starts with $\delta=0$. If a feasible solution cannot be obtained, the value of $\delta$ is increased in $\Delta \delta=0.1$ and the procedure starts over again. It ends when a feasible solution is obtained.

\subsubsection{Suppliers clustering}

The previous procedure provides the subset of vehicles, $R^{\prime} \subseteq R$, available to serve the demand. To obtain a feasible solution using this number of vehicles, the suppliers are clustered and served by one of these vehicles, as shown in Fig. 3. The assignment is made considering the proximity criterion by preserving feasibility in terms of capacity. Then, the supplier $p_{j}$ is assigned to the closest vehicle that can satisfy its demand. If more than one vehicle is located at the same distance from $p_{j}$, the supplier is assigned to the vehicle with the highest free capacity. If the tie persists, the vehicle that serves $p_{j}$ is selected arbitrarily. The route for each vehicle is built by visiting suppliers in the same order as they are included during the assignment procedure.

Note that, even when the artificial demand is considered, it is possible to obtain an infeasible solution. When this happens, the value of $\delta$ is increased and a new subset of vehicles is selected with the purpose of constructing new clusters of suppliers. 


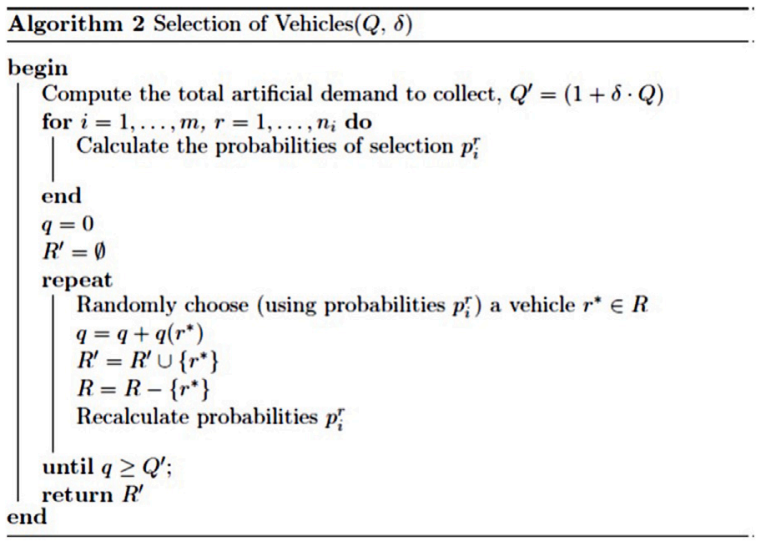

Fig. 2. Procedure for the selection of vehicles.

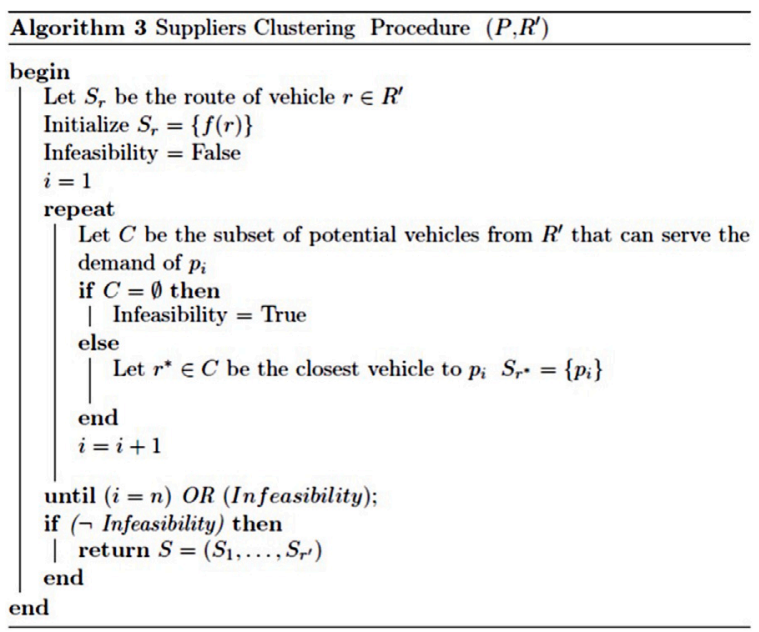

Fig. 3. Procedure for the assignment of suppliers to vehicles.

\subsection{Improvement phase}

Keeping in mind that the constructive procedure previously described can generate low quality initial solutions, the algorithm incorporates an improvement procedure based on local search. Two local searches are considered: (1) intra-route local search, which applies movements that only affects the sequence in a single route; (2) inter-routes local search, which involves movements affecting suppliers belonging to different routes. Both local searches incorporate two types of movements: interchange and reallocation.

The objective of the intra-route procedure is to reallocate suppliers inside each route separately, while the inter-routes movement searches for reallocating suppliers from different routes. Note that the intraroute movements are always feasible since the route is feasible in terms of capacity. However, this is not always possible for the inter-route movements.

Next, the moves of $2-o p t$, interchange and reallocation employed by both local searches are explained.

- Intra-route 2-opt. Two edges are deleted from the current route and paths are reconnected in a different way, obtaining a new route.

- Intra-route interchange. Given two suppliers belonging to the same route, their positions are interchanged into the route. If the suppliers belong to positions $i$ and $j$, arcs $(i-1, i),(i, i+1),(j-1, j)$ and $(j, j+1)$ are replaced by $\operatorname{arcs}(i-1, j),(j, i+1),(j-1, i)$ and

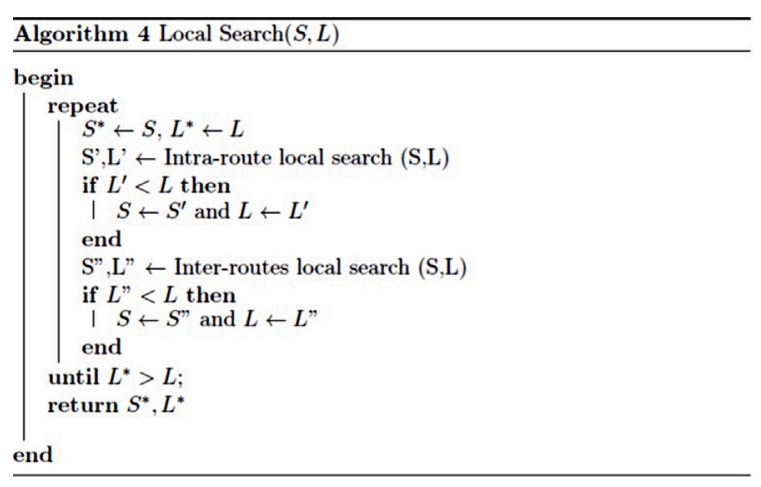

Fig. 4. Improvement Phase.

$(i, j+1)$. It is important to remark that these movements are always feasible in terms of capacity.

- Intra-route reallocation. For a given supplier, its best position inside of the route is identified. If the best identified position is different to the current one, the movement is performed.

- Inter-routes interchange. Given two suppliers belonging to different routes, they are interchanged as long as the improvement move keeps feasibility (in terms of capacity).

- Inter-routes reallocation. For a given supplier, its best position is identified in any of the remaining routes, i.e., an exhaustive search is performed through all of the available positions in the other routes to find the position in which the minimum increase is produced. If the new best position is identified, i.e. the global objective value is improved, the movement is performed.

Both local searches are applied hierarchically. Initial solutions are improved by applying the intra-local search procedure first, starting with the 2-opt. Next, the obtained local minima is improved by applying the inter-routes local search (starting with the inter-routes interchange). These procedures are iteratively applied while the current solution value $L$ keeps improving. In each procedure, the reallocation movement is performed first and the interchange movement is executed next. Fig. 4 exhibits this process.

\section{Computational experiments}

In this section, the experimental results are reported. First, the details for the results of the case of study are presented. Next, the current solution implemented by the company is used to compare with the results obtained by both, the mathematical model and the algorithm proposed.

A second analysis studies the performance of the metaheuristic compared to the model solved using commercial optimization software, in instances obtained by variations of the data of the company. These variations involved an increase in the number of the suppliers and variations of their demands. In this additional experimentation two indicators were used: (1) efficiency regarding the quality of the solution and (2) size of the instances (regarding the number of nodes) solved in a reasonable amount of time. A third analysis was conducted by modifying benchmark instances, and solving them using the mathematical model and the algorithm. The limits of the implementation of the model were determined in terms of the size of the instances that can be solved optimally in a time limit of two hours. Regarding the performance of the algorithm, it was evaluated in terms of the quality of the best solutions found. Sections 6.5.2 and 6.5.3 discuss the results obtained.

Finally, experimentation considering the set of instances proposed for the MDOVRP (the most similar problem identified in the literature) was used to assess the performance of the proposed approaches with respect to the best-known results (BKR) reported in the literature. 
Table 1

Suppliers information.

\begin{tabular}{llllr}
\hline Supplier code & City & $x_{-}$coordinate & $y_{-}$coordinate & $\begin{array}{c}\text { Units to collect } \\
\text { (pallets) }\end{array}$ \\
\hline$P_{1}$ & Manchester & 42.90959 & -89.20563 & 10 \\
$P_{2}$ & Bardstown & 44.45500 & -89.49700 & 8 \\
$P_{3}$ & Chicago & 42.02182 & -88.32232 & 15 \\
$P_{4}$ & Endeavor & 41.70834 & -87.79828 & 11 \\
$P_{5}$ & Hackensack & 38.15349 & -85.68189 & 13 \\
$P_{6}$ & Duluth & 40.95986 & -81.46269 & 9 \\
$P_{7}$ & Copley & 38.09182 & -84.51974 & 10 \\
$P_{8}$ & Boston & 33.96039 & -84.08368 & 12 \\
$P_{9}$ & Milwakee & 33.41514 & -84.74180 & 9 \\
$P_{10}$ & Asheboro & 36.13115 & -80.08082 & 14 \\
$P_{11}$ & Fairburn & 40.83432 & -74.05586 & 12 \\
$P_{12}$ & New Berlin & 41.57194 & -72.77388 & 8 \\
$P_{13}$ & Washington & 42.26526 & -71.69248 & 12 \\
\hline
\end{tabular}

All of the experiments were executed using an Intel Core i5-6300 @ $2.40 \mathrm{GHz} 8 \mathrm{~GB}$ RAM PC under Windows 10 . The mathematical model was implemented in the AMPL optimization language and solved with CPLEX 12.6.0. Regarding the algorithm, it was coded in C++ under Visual Studio 2015.

\subsection{Case of study}

As previously described, the case of study is inspired on a company that uses a single carrier to collect raw material from different suppliers located in United States. This document analyze the case if, in stead of a single carrier, several carriers are hired for the transportation of raw material to the delivery point. The interest raised because of the high transportation costs of having a single carrier. In order to do this, they identified the potential carriers that may provide the service, their location, their available fleet and the costs that will be charged. The objective is to compare the costs charged by the current company, with the costs of working with several transportation companies.

To generate an instance that represents a typical workday, the coordinates of the suppliers and the potential carriers are considered. In total, 13 suppliers and 13 carriers are included. For each carrier, up to 4 different vehicles per carrier are considered. As a result, up to 41 vehicles are available. To maintain confidentiality of the data, the information is disguised.

Tables 1 and 2 describe the information used to configure the instance. In Table 1, the first column displays the label of the instance and column 2 indicates the name of the city. Columns 3, 4 and 5 indicate the coordinates $(x, y)$ and the units to collect, respectively. On the other hand, in Table 2, the first column also indicates the label of the instance, and column 2 the name of the city. Columns 3, and 4 display the coordinates $(x, y)$ while columns 5,6 and 7 show the details for the number of available vehicles, vehicles' capacities and contracting fixed costs. The carriers $o_{1}, o_{2}, \ldots, o_{13}$ are located in the following cities, respectively: Stoughton-Madison, Stevens Point, Elgin, Bridgeview, Louisville, Akron-Canton, Lexington, Norcross, Newman, Winston, Carlstadt, Hartford and Worcester.

The delivery point (border customs) is located in Carretera Nuevo Laredo - Piedras Negras km. 12.5, Puente Comercio Mundial Sector Centro, 88000. Nuevo Laredo, Tamaulipas, México, with coordinates (27.595137, -99.544912).

\subsection{New scenarios for the case of study}

From the data provided by the company, we created new instances by varying the demands of the suppliers. As result, 15 new instances simulating workday scenarios were created. Table 3 displays the demand of each supplier in accordance with each scenario. Column 1 shows the code of the scenario, and the headers of the columns exhibits the code of the supplier. The numbers into the table represent the units
Table 3

New scenarios.

\begin{tabular}{rrrrrrrrrrrrrr}
\hline & $p_{1}$ & $p_{2}$ & $p_{3}$ & $p_{4}$ & \multicolumn{1}{c}{$p_{5}$} & $p_{6}$ & $p_{7}$ & $p_{8}$ & $p_{9}$ & $p_{10}$ & $p_{11}$ & $p_{12}$ & $p_{13}$ \\
\hline$I_{1}$ & 12 & 14 & 11 & 10 & 10 & 8 & 9 & 0 & 14 & 12 & 14 & 13 & 10 \\
$I_{2}$ & 10 & 0 & 10 & 13 & 9 & 13 & 12 & 23 & 14 & 15 & 10 & 15 & 0 \\
$I_{3}$ & 9 & 8 & 14 & 0 & 10 & 9 & 11 & 13 & 0 & 15 & 9 & 9 & 0 \\
$I_{4}$ & 10 & 13 & 0 & 0 & 8 & 15 & 9 & 0 & 14 & 11 & 15 & 8 & 11 \\
$I_{5}$ & 10 & 10 & 0 & 13 & 11 & 12 & 13 & 0 & 13 & 11 & 12 & 13 & 9 \\
$I_{6}$ & 0 & 11 & 13 & 0 & 9 & 12 & 10 & 13 & 19 & 15 & 13 & 0 & 10 \\
$I_{7}$ & 9 & 0 & 14 & 0 & 13 & 9 & 15 & 11 & 0 & 8 & 12 & 11 & 14 \\
$I_{8}$ & 0 & 21 & 0 & 14 & 0 & 13 & 10 & 0 & 14 & 12 & 19 & 0 & 0 \\
$I_{9}$ & 9 & 0 & 14 & 0 & 13 & 9 & 15 & 11 & 0 & 8 & 12 & 11 & 14 \\
$I_{10}$ & 9 & 14 & 11 & 13 & 14 & 15 & 12 & 13 & 8 & 9 & 13 & 14 & 15 \\
$I_{11}$ & 0 & 13 & 11 & 0 & 10 & 20 & 11 & 13 & 0 & 11 & 15 & 13 & 0 \\
$I_{12}$ & 8 & 15 & 11 & 14 & 12 & 10 & 13 & 14 & 0 & 10 & 8 & 18 & 9 \\
$I_{13}$ & 12 & 0 & 10 & 15 & 14 & 0 & 15 & 14 & 8 & 15 & 9 & 11 & 10 \\
$I_{14}$ & 14 & 13 & 10 & 14 & 14 & 12 & 15 & 13 & 12 & 13 & 14 & 0 & 10 \\
$I_{15}$ & 15 & 15 & 12 & 9 & 14 & 0 & 24 & 18 & 8 & 9 & 0 & 10 & 13 \\
\hline
\end{tabular}

to be collected from each supplier, in number of pallets. A value of zero means that the supplier has no units to be collected, and shall be omitted in the routing. For instance, in scenario $I_{8}$ raw material of 7 suppliers must be collected $\left(p_{2}, p_{4}, p_{6}, p_{7}, p_{9}, p_{10}, p_{11}\right)$ by selecting vehicles from the 13 carriers available.

\subsection{Set of additional instances}

To validate the effectiveness of the model and the efficiency of the metaheuristic, benchmark instances from the literature were adapted. To facilitate the modification, these test instances were extracted from those available for the multi-depot vehicle routing problem. These instances also consider the fact that from each depot, more than one vehicle is available. Finally, one of the nodes belonging to the "customers" set, is defined as the delivery point.

The instances used to conduct the additional experimentation were obtained from Koulaeian et al. (2015) (Kou15), Chunyu and Xiaobo (2010) (CaX10), Wang and Wu (2015) (WaW15), Gillett and Miller (1974) (GaMG74) and Gillett and Johnson (1976) (GaJ76-7 to 12), since they show a close relationship with the purpose of this research. The adaption consists of selecting one of the nodes from the customers set as the delivery point, and defining the depot (carrier) nodes as the potential origins. In each origin, both the contracting costs and vehicle capacities were diversified. These instances were useful because they also consider a heterogeneous fleet. Information about the fleet is displayed in Table 4.

\subsection{Benchmark MDOVRP instances}

The set of instances proposed by Cordeau et al. (1997) were tested, but specific adjustments were made to both the formulation and the algorithm for making them able to deal with the MDOVRP. It is important to remark that, for this analysis, we are only interested in comparing the performance in terms of the quality of the solutions, leaving aside the discussion of the elapsed times, since our approaches are not specifically designed for the MDOVRP.

\subsection{Experimental results}

This section is devoted to reporting the computational experiments carried out for assessing the performance of the mathematical model and the computational algorithm proposed in this work. Regarding the model, a time limit of two hours (7200 s) was set for CPLEX to solve each instance. For the cases in which the model reached the time limit, the best solution obtained so far is reported. The metaheuristic was executed 15 times, and the results displayed correspond to the best solution found and the average CPU time. 


\subsubsection{Case of study}

Fig. 5 illustrates the solution given by the logistic planner of the company for the workday used in the case of study. Four vehicles were used, incurring in a total cost of 133.8611. The planner justified this solution by the fact that the contracting cost is minimum and the arrival time to the final destination is "short". It can be observed that 3 routes are balanced (in terms of total cost) and the other one is lightly superior.

Both, the formulation and the metaheuristic procedure, were capable to solve the case study. In particular, the metaheuristic algorithm was also able to find the optimal solution. The solution obtained is exhibited in Fig. 6. Two vehicles were employed, representing a total cost of 92.5384 . That is, it produced savings of $30.86 \%$.

It is important to remark that the optimal solution was obtained in a significantly short time $(2.64 \mathrm{~s})$, and the metaheuristic found the optimal solution even in a shorter time (0.113 s).

\subsubsection{New scenarios}

Table 5 reports the results obtained by both the model and the algorithm for the scenarios shown in Table 3. The first three columns of Table 5 show the name of the instance, number of suppliers and number of carriers available, respectively. In these scenarios derived from the data provided by the manufacturing company, it is considered that the vehicles available for each carrier have the characteristics shown in Table 2. As indicated above, only the orders of the suppliers were modified. The next three columns show the optimal objective value, the number of vehicles chosen and the time (seconds) required by CPLEX to solve the model. Columns 7, 8 and 9 show the value of the best solution obtained by the metaheuristic, the number of vehicles selected in this solution and the execution time (seconds). The last column presents the percentage of improvement of the metaheuristic respect to the solutions obtained by the model, calculated as shown in Eq. (16):

$$
\text { Percentage of improvement }=\frac{100 \cdot\left(\text { Model } f^{*}-\text { Metaheuristic } f^{\text {best }}\right)}{\text { Model } f^{*}}
$$

As it can be observed, the algorithm was able to find the optimal solution for 12 out of 14 instances.

Table 6 exhibits the statistics for the objective function of the solutions obtained with the metaheuristic algorithm, for all the runs executed. The first column shows the ID of the instance. The second and third columns present the results of the average and standard deviation of the objective function, respectively. The third column calculates the coefficient of variation, i.e. the standard deviation divided by the average. Columns 4, 5 and 6 show the median, minimum and maximum values. In the seventh column, the objective function obtained with the model is presented, which is the same value shown in column 4 of Table 5. A statistical non-parametric test of signs was conducted to determine if the median of the objective function obtained with the metaheuristic is equal to the objective function obtained with the model. This test was selected because of the small number of runs executed, and the assumption of a distribution biased to the optimal value. The test was executed in Minitab, and the resulting p-values are shown in the eight column of the table. It can be observed that the coefficient of variation is below $11 \%$ in general, which is interpreted as a low variation of the results respect to the metaheuristic. Finally, the p-values allows concluding that, with a significance level of 0.05 , the median values of the metaheuristic are not equal to those obtained with the model in all of the instances. Although the optimal values are found with the algorithm, the variation of the results pushes up the median values. Considering the computational effort required by the algorithm to solve these small instances, it can be run many times and eventually the optimal value will be found.

\subsubsection{Additional benchmark modified instances}

To validate the effectiveness of both, the formulation and the algorithm, the set of additional instances was solved. Table 7 reports these results. The first four columns of Table 7 display the name of the instance, number of suppliers, number of carriers (depots) available, and the number of available vehicles respectively. The next three columns show the number of vehicles chosen in the optimal solution, the optimal objective value, and the time (seconds) required by CPLEX for the solution of the model, respectively. Columns 8, 9, and 10 indicate for the metaheuristic, the number of vehicles chosen, the best objective value, and the time (seconds) required for the execution. The last column presents the percentage of improvement of the metaheuristic respect to the solutions obtained by the model, calculated as shown in Eq. (16).

As it can be seen in Table 7, the metaheuristic shows a good behavior. For nine out of the ten instances, it provides solutions equal to or better than those found by the commercial solver. In the other instances, the difference is $1.010 \%$. On the other hand, it must be underlined that, for the instances GaJ76-7, GaJ76-8, GaJ76-9, GaJ76-10, GaJ76-11 and GaJ76-12, the commercial solver reached the maximum time allowed. In all of these cases, the metaheuristic reported solutions that improved up to $14.683 \%$ the solution reported by the solver, spending around 7 seconds. The average metaheuristic execution time was $9.333 \mathrm{~s}$ versus to the $4690.307 \mathrm{~s}$ required by the solver. This means a reduction of $99.8 \%$.

Table 8 exhibits the statistics for the objective function of the solutions obtained with the metaheuristic algorithm, for all the runs executed, for the additional instances. The first column shows the ID of the instance. The second and third column present the results of the average and standard deviation of the objective function, respectively. The third column calculates the coefficient of variation, i.e. the standard deviation divided by the average. Columns 4, 5 and 6 show the median, minimum and maximum values. In the seventh column, the objective function obtained with the model is presented, which is the same value shown in column 6 of Table 7. A statistical non-parametric

Table 2

\begin{tabular}{|c|c|c|c|c|c|c|}
\hline $\begin{array}{l}\text { Carrier } \\
\text { code }\end{array}$ & City & $x_{-}$coordinate & $y_{-}$coordinate & $\begin{array}{l}\text { Number of } \\
\text { vehicles }\end{array}$ & Capacities & $\begin{array}{l}\text { Contracting } \\
\text { costs }\end{array}$ \\
\hline$O_{1}$ & Stoughton - Madison & 41.795667 & -72.581040 & 3 & $45,60,75$ & $7.5,12,15$ \\
\hline $\mathrm{O}_{3}$ & Elgin & 42.111290 & -88.060750 & 3 & $40,45,55$ & $7,8.5,12.5$ \\
\hline $\mathrm{O}_{4}$ & Bridgeview & 43.708506 & -89.470503 & 2 & 45,55 & 9,12 \\
\hline $\mathrm{O}_{7}$ & Lexington & 41.100610 & -81.652772 & 3 & $45,50,60$ & $9,10,11.5$ \\
\hline$O_{8}$ & Norcross & 42.414253 & -71.684454 & 3 & $60,65,75$ & $12,13.5,15$ \\
\hline$O_{9}$ & Newman & 43.116437 & -89.660384 & 3 & $45,45,55$ & $8,8,9.5$ \\
\hline$O_{10}$ & Winston & 35.678212 & -79.857301 & 4 & $50,60,65,70$ & $10,12,13,14$ \\
\hline$O_{11}$ & Carlstadt & 33.554384 & -84.593951 & 2 & 40,70 & 7,13 \\
\hline$O_{12}$ & Hartford & 41.794759 & -87.758490 & 4 & $40,50,70,75$ & $7,9,13,15$ \\
\hline
\end{tabular}


Table 4

Additional modified benchmark instances.

\begin{tabular}{|c|c|c|c|c|}
\hline $\begin{array}{l}\text { Instance } \\
\text { name }\end{array}$ & origin & $\begin{array}{l}\text { Number of } \\
\text { vehicles }\end{array}$ & Capacities & Contracting costs \\
\hline \multirow{3}{*}{ Kou15 } & $O_{1}$ & 2 & 400,400 & 22,22 \\
\hline & $\mathrm{O}_{2}$ & 4 & $200,200,200,200$ & $11,11,11,11$ \\
\hline & $\mathrm{O}_{3}$ & 2 & 400,400 & 22,22 \\
\hline \multirow{2}{*}{ CaX10 } & $O_{1}$ & 3 & $8,6,4$ & $3,2,1$ \\
\hline & $\mathrm{O}_{2}$ & 3 & $8,6,4$ & $3,2,1$ \\
\hline \multirow{3}{*}{ WaW15 } & $O_{1}$ & 4 & $20,20,15,15$ & $5,5,4,4$ \\
\hline & $\mathrm{O}_{2}$ & 5 & $20,20,15,15,15$ & $5,5,4,4,4$ \\
\hline & $\mathrm{O}_{3}$ & 6 & $20,20,20,15,15,15$ & $5,5,5,4,4,4$ \\
\hline \multirow{3}{*}{ GaMG74 } & $O_{1}$ & 6 & $30,40,50,50,75,75$ & $2522.266,2898.474,3202.73,3202.73,3648.58,3648.58$ \\
\hline & $\mathrm{O}_{2}$ & 6 & $8,8,18,20,40,75$ & $1412.7235,1412.7235,1950.8235,2074.106,2898.474,3648.58$ \\
\hline & $\mathrm{O}_{3}$ & 6 & $20,30,40,50,50,75$ & $2074.106,2522.266,2898.474,3202.73,3202.73,3648.58$ \\
\hline \multirow{4}{*}{ GaJ76-7 } & $O_{1}$ & 4 & $55,60,65,75$ & $3327.876,3435.034,3524.204,3648.58$ \\
\hline & $\mathrm{O}_{2}$ & 4 & $8,20,40,50$ & $1412.7235,2074.106,2898.474,3202.73$ \\
\hline & $\mathrm{O}_{3}$ & 4 & $10,30,50,75$ & $1553.994,2522.266,3202.73,3648.58$ \\
\hline & $\mathrm{O}_{4}$ & 4 & $75,75,75,75$ & $3648.58,3648.58,3648.58,3648.58$ \\
\hline \multirow{2}{*}{ GaJ76-8 } & $O_{1}$ & 7 & $30,30,40,50,50,75,75$ & $2522.266,2522.266,2898.474,3202.73,3202.73,3648.58,3648.58$ \\
\hline & $\mathrm{O}_{2}$ & 7 & $50,50,50,75,75,75,75$ & $3202.73,3202.73,3202.73,3648.58,3648.58,3648.58,3648.58$ \\
\hline \multirow{3}{*}{ GaJ76-9 } & $O_{1}$ & 10 & $8,8,18,18,30,30,40,40,50,50$ & $1412.7235,1412.7235,1950.8235,1950.8235,2522.266,2522.266,2898.47$ \\
\hline & $\mathrm{O}_{2}$ & 10 & $8,20,20,50,50,75,75,75,75,75$ & $1412.7235,2074.106,2074.106,3202.73,3202.73,3648.58,3648.58,3648.58,3648.58,3648.58$ \\
\hline & $\mathrm{O}_{3}$ & 10 & $18,18,50,50,50,75,75,75,75,75$ & $1950.8235,1950.8235,3202.73,3202.73,3202.73,3648.58,3648.58,3648.58,3648.58,3648.58$ \\
\hline \multirow{5}{*}{ GaJ76-10 } & $O_{1}$ & 8 & $50,50,75,75,75,75,75,75$ & $3202.73,3202.73,3648.58,3648.58,3648.58,3648.58,3648.58,3648.58$ \\
\hline & $\mathrm{O}_{2}$ & 8 & $8,8,18,18,30,30,40,40$ & $1412.7235,1412.7235,1950.8235,1950.8235,2522.266,2522.266,2898.474,2898.474$ \\
\hline & $\mathrm{O}_{3}$ & 8 & $18,18,50,50,50,75,75,75$ & $1950.8235,1950.8235,3202.73,3202.73,3202.73,3648.58,3648.58,3648.5$ \\
\hline & $\mathrm{O}_{4}$ & 8 & $8,20,20,50,50,75,75,75$ & $1412.7235,2074.106,2074.106,3202.73,3202.73,3648.58,3648.58,3648.58$ \\
\hline & $O_{5}$ & 8 & $30,30,40,40,50,50,75,75$ & $2522.266,2522.266,2898.474,2898.474,3202.73,3202.73,3648.58,3648.58$ \\
\hline \multirow{4}{*}{ GaJ76-11 } & $O_{1}$ & 8 & $50,50,75,75,75,75,75,75$ & $3202.73,3202.73,3648.58,3648.58,3648.58,3648.58,3648.58,3648.58$ \\
\hline & $\mathrm{O}_{2}$ & 8 & $8,8,18,18,30,30,40,40$ & $1412.7235,1412.7235,1950.8235,1950.8235,2522.266,2522.266,2898.474,2898.474$ \\
\hline & $\mathrm{O}_{3}$ & 8 & $18,18,50,50,50,75,75,75$ & $1950.8235,1950.8235,3202.73,3202.73,3202.73,3648.58,3648.58,3648.58$ \\
\hline & $\mathrm{O}_{4}$ & 8 & $8,20,20,50,50,75,75,75$ & $1412.7235,2074.106,2074.106,3202.73,3202.73,3648.58,3648.58 .3648 .58$ \\
\hline \multirow{4}{*}{ GaJ76-12 } & $O_{1}$ & 8 & $8,8,18,18,30,30,40,40$ & $1412.7235,1412.7235,1950.8235,1950.8235,2522.266,2522.266,2898.474$ \\
\hline & $\mathrm{O}_{2}$ & 8 & $8,20,20,50,50,75,75,75$ & 1412.7235, 2074.106, 2074.106, 3202.73, 3202.73, 3648.58, 3648.58, 3648.58 \\
\hline & $\mathrm{O}_{3}$ & 8 & $18,18,50,50,50,75,75,75$ & $1950.8235,1950.8235,3202.73,3202.73,3202.73,3648.58,3648.58,3648.58$ \\
\hline & $\mathrm{O}_{4}$ & 8 & $50,50,75,75,75,75,75,75$ & $3202.73,3202.73,3648.58,3648.58,3648.58,3648.58,3648.58,3648.58$ \\
\hline
\end{tabular}

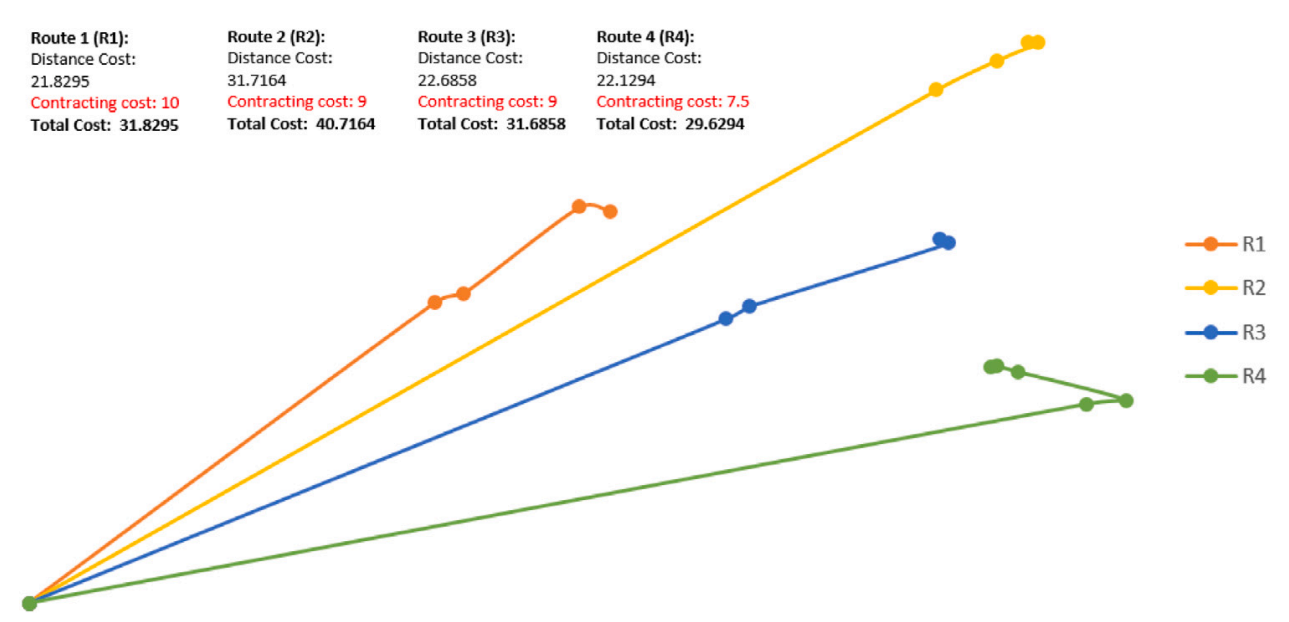

Fig. 5. Diagram of the Current Situation for the case of study.

test of signs was applied to determine if the median of the objective function obtained with the metaheuristic is less than or equal to the objective function obtained with the model. This test was selected because of the small number of runs executed, and the assumption of a distribution biased to the optimal value. The test was executed in Minitab, and the resulting p-values are shown in the eight column of the table. It can be observed that the coefficient of variation is below $7 \%$ for the smaller instances, and below $4 \%$ for the larger instances, which are interpreted as a low variation of the results obtained with the metaheuristic. Finally, the p-values allows concluding that, with a significance level of 0.05 , the median values of the metaheuristic are less than or equal to those obtained with the model in 5 out of 10 instances. Comparing these results with those obtained in Table 6 it becomes evident that the algorithm is efficient to find better solutions than the model solved with the commercial solver for large instances, and its efficiency is lower for small instances.

\subsection{Experimentation using MDOVRP benchmark instances}

As explained above, the problem addressed in this research is new respect to the literature. Thus, our approaches are evaluated on the MDOVRP and compared with the best-known results published in 


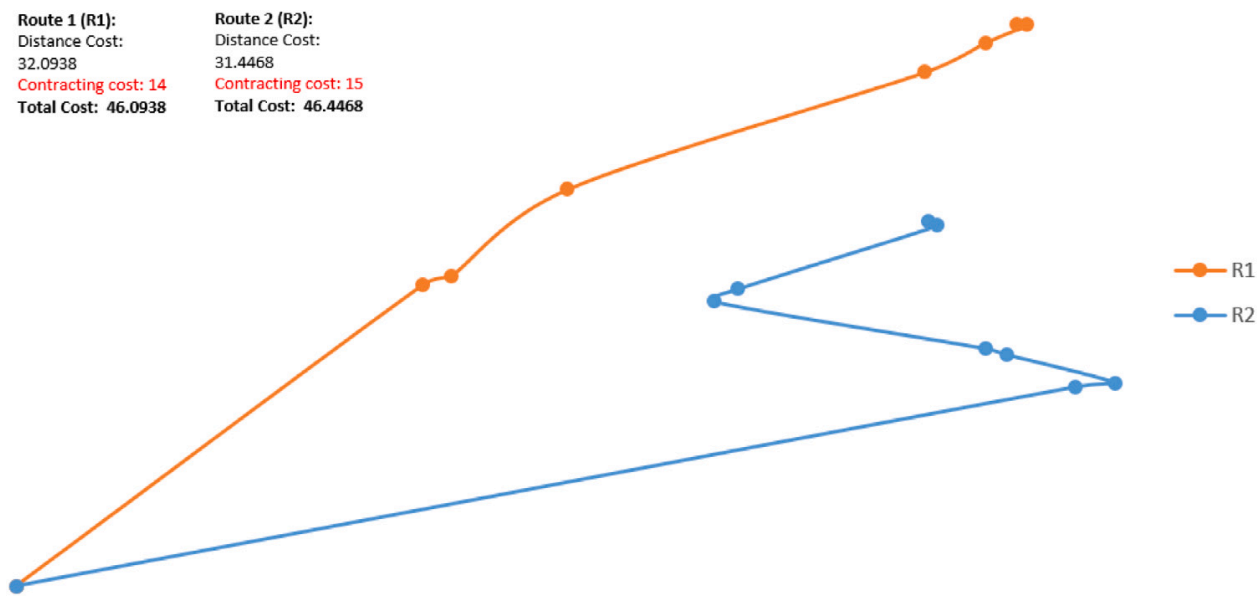

Fig. 6. Optimal Solution for the case of study.

Table 5

Results obtained by the formulation and metaheuristic algorithm for the case study instances.

\begin{tabular}{|c|c|c|c|c|c|c|c|c|c|}
\hline & \multirow[t]{2}{*}{$|P|$} & \multirow[t]{2}{*}{$|O|$} & \multicolumn{3}{|l|}{ Model } & \multicolumn{3}{|c|}{ Metaheuristic } & \multirow{2}{*}{$\begin{array}{l}\text { Percentage of } \\
\text { improvement }\end{array}$} \\
\hline & & & $f^{*}$ & $k$ & Time & $f^{\text {best }}$ & $k$ & Time & \\
\hline$I_{2}$ & 12 & 13 & 92.163 & 2 & 2.000 & 92.163 & 2 & 0.155 & 0.000 \\
\hline$I_{3}$ & 11 & 13 & 95.081 & 2 & 1.938 & 95.589 & 2 & 0.161 & -0.534 \\
\hline$I_{4}$ & 10 & 13 & 84.385 & 2 & 3.531 & 84.385 & 2 & 0.132 & 0.000 \\
\hline$I_{5}$ & 10 & 13 & 87.138 & 2 & 2.141 & 87.138 & 2 & 0.115 & 0.000 \\
\hline$I_{6}$ & 11 & 13 & 89.589 & 2 & 1.578 & 89.589 & 2 & 0.125 & 0.000 \\
\hline$I_{7}$ & 10 & 13 & 89.666 & 2 & 0.797 & 89.666 & 2 & 0.117 & 0.000 \\
\hline$I_{8}$ & 10 & 13 & 86.397 & 2 & 2.688 & 86.397 & 2 & 0.100 & 0.000 \\
\hline$I_{9}$ & 7 & 13 & 75.740 & 2 & 3.125 & 75.847 & 2 & 0.069 & -0.141 \\
\hline$I_{10}$ & 10 & 13 & 86.397 & 2 & 2.500 & 86.397 & 2 & 0.105 & 0.000 \\
\hline$I_{11}$ & 13 & 13 & 114.216 & 3 & 14.094 & 114.216 & 3 & 0.194 & 0.000 \\
\hline$I_{12}$ & 9 & 13 & 86.384 & 2 & 1.328 & 86.384 & 2 & 0.104 & 0.000 \\
\hline$I_{13}$ & 11 & 13 & 92.655 & 2 & 2.719 & 92.655 & 2 & 0.168 & 0.000 \\
\hline$I_{14}$ & 12 & 13 & 94.959 & 2 & 1.328 & 94.959 & 2 & 0.143 & 0.000 \\
\hline$I_{15}$ & 11 & 13 & 91.857 & 2 & 9.859 & 91.857 & 2 & 0.129 & 0.000 \\
\hline \multicolumn{3}{|c|}{ Average } & & & 3.545 & & & 0.130 & -0.048 \\
\hline
\end{tabular}

Table 6

Statistics of the objective function obtained with the metaheuristic for the case study instances.

\begin{tabular}{|c|c|c|c|c|c|c|c|c|}
\hline & $f_{\text {ave }}$ & $f_{\text {stdev }}$ & $f_{c o v}$ & $f_{\text {median }}$ & $f_{\min }$ & $f_{\max }$ & $f_{\text {model }}$ & $p$-value \\
\hline$I_{2}$ & 103.883 & 10.902 & 0.105 & 108.287 & 92.163 & 119.571 & 92.163 & 0.000 \\
\hline$I_{3}$ & 105.923 & 6.931 & 0.065 & 107.912 & 95.589 & 117.208 & 95.081 & 0.000 \\
\hline$I_{4}$ & 90.289 & 9.480 & 0.105 & 86.385 & 84.385 & 108.956 & 84.385 & 0.004 \\
\hline$I_{5}$ & 92.686 & 8.915 & 0.096 & 88.724 & 87.138 & 109.709 & 87.138 & 0.004 \\
\hline$I_{6}$ & 97.217 & 9.870 & 0.102 & 92.597 & 89.589 & 114.523 & 89.589 & 0.001 \\
\hline$I_{7}$ & 98.350 & 10.376 & 0.105 & 91.666 & 89.666 & 113.346 & 89.666 & 0.004 \\
\hline$I_{8}$ & 92.055 & 9.854 & 0.107 & 87.251 & 86.397 & 111.094 & 86.397 & 0.004 \\
\hline$I_{9}$ & 77.965 & 4.967 & 0.064 & 76.399 & 75.847 & 95.268 & 75.740 & 0.000 \\
\hline$I_{10}$ & 87.196 & 1.127 & 0.013 & 86.397 & 86.397 & 89.251 & 86.397 & 0.016 \\
\hline$I_{11}$ & 117.458 & 7.189 & 0.061 & 115.266 & 114.216 & 142.881 & 114.216 & 0.001 \\
\hline$I_{12}$ & 91.335 & 7.882 & 0.086 & 87.878 & 86.384 & 109.863 & 86.384 & 0.004 \\
\hline$I_{13}$ & 102.941 & 9.384 & 0.091 & 108.671 & 92.655 & 117.816 & 92.655 & 0.000 \\
\hline$I_{14}$ & 103.393 & 9.300 & 0.090 & 97.933 & 94.959 & 120.836 & 94.959 & 0.008 \\
\hline$I_{15}$ & 100.67 & 9.778 & 0.097 & 91.857 & 91.857 & 112.482 & 91.857 & 0.016 \\
\hline
\end{tabular}

the literature. It is important to remark that we have to adapt our formulation and algorithm to make them suitable to solve that particular problem. The modifications made and the obtained results are described next.

\subsubsection{Model results}

Our proposed model will be compared with the ones presented in Lalla-Ruiz et al. (2016). Before implementing our formulation to the set of benchmark instances, we made the following adjustments: (1) we removed the constraint that forces having a common final destination, (2) we also relaxed the restrictions that establish a maximum fleet size per origin. (3) we set the contracting cost as zero since these instances do not consider any contracting cost. It is important to notice that, even when we could have relaxed the constraints related to the capacity of the vehicle, avoiding the use of index $r$ in variables $o_{i j}^{r}$ and $v_{i j}^{r}$, we did not want to do that so we can implement the model without losing generality. Additionally, we maintain the 2-hours time limit established for previous experiments.

The results are compared with the best known results published in Lalla-Ruiz et al. (2016), who also implemented and used the formulation proposed in Liu et al. (2014). Even when a recent publication (Brandão, 2020) outperformed these results, we decided not to 
Table 7

Results obtained by the formulation and the algorithm for the set of additional instances.

\begin{tabular}{|c|c|c|c|c|c|c|c|c|c|c|}
\hline \multirow[t]{2}{*}{ Instance } & \multirow[t]{2}{*}{$|P|$} & \multirow[t]{2}{*}{$|O|$} & \multirow{2}{*}{$\begin{array}{l}\text { Total available } \\
\text { vehicles }\end{array}$} & \multicolumn{3}{|c|}{ Model } & \multicolumn{3}{|c|}{ Metaheuristic } & \multirow{2}{*}{$\begin{array}{l}\text { Percentage of } \\
\text { improvement }\end{array}$} \\
\hline & & & & $k$ & $f^{*}$ & Time & $k$ & $f^{\text {best }}$ & Time & \\
\hline Kou15 & 12 & 3 & 8 & 2 & 2903.525 & 0.62 & 5 & 2903.525 & 1.455 & 0.000 \\
\hline Cax10 & 20 & 2 & 6 & 4 & 939.919 & 1.467 & 4 & 939.919 & 0.157 & 0.000 \\
\hline WaW15 & 25 & 3 & 15 & 7 & 689.067 & 29.484 & 7 & 689.067 & 1.384 & 0.000 \\
\hline GaMG74 & 29 & 3 & 18 & 8 & 26832.365 & 3671.500 & 8 & 27103.400 & 1.389 & -1.010 \\
\hline GaJ76-7 & 49 & 4 & 16 & 16 & 51185.302 & 7200.000 & 13 & 43669.900 & 7.439 & 14.683 \\
\hline GaJ76-8 & 49 & 2 & 14 & 6 & 47270.60 & 7200.000 & 6 & 47253.400 & 15.835 & 0.036 \\
\hline GaJ76-9 & 74 & 3 & 30 & 10 & 37349.500 & 7200.000 & 10 & 33855.500 & 10.793 & 9.355 \\
\hline GaJ76-11 & 74 & 4 & 32 & 10 & 37187.100 & 7200.000 & 8 & 33352.100 & 9.909 & 10.313 \\
\hline GaJ76-12 & 74 & 4 & 32 & 10 & 37174.675 & 7200.000 & 10 & 33343.800 & 9.460 & 10.305 \\
\hline GaJ76-10 & 99 & 5 & 40 & 24 & 92565.734 & 7200.000 & 31 & 89885.700 & 35.508 & 2.895 \\
\hline Average & & & & & & 4690.307 & & & 9.333 & 4.658 \\
\hline
\end{tabular}

Table 8

Statistics of the objective function obtained with the metaheuristic for the set of additional instances.

\begin{tabular}{|c|c|c|c|c|c|c|c|c|}
\hline & $f_{\text {ave }}$ & $f_{\text {stdev }}$ & $f_{c o v}$ & $f_{\text {median }}$ & $f_{\min }$ & $f_{\max }$ & $f_{\text {model }}$ & $p$-value \\
\hline Kou15 & 3228.564 & 204.016 & 0.063 & 3218.580 & 2903.525 & 3557.170 & 2903.525 & 0.004 \\
\hline CaX10 & 942.912 & 2.898 & 0.003 & 945.530 & 939.919 & 945.530 & 939.919 & 0.004 \\
\hline WaW15 & 702.012 & 6.910 & 0.010 & 702.609 & 689.067 & 713.465 & 689.067 & 0.000 \\
\hline GaMG74 & 28319.047 & 1252.052 & 0.044 & 27952.900 & 27103.400 & 30296.700 & 26832.365 & 0.000 \\
\hline GaJ76-7 & 44460.553 & 219.205 & 0.005 & 44516.800 & 43669.900 & 44539.200 & 51185.302 & 1.000 \\
\hline GaJ76-8 & 47297.247 & 13.650 & 0.000 & 47299.900 & 47253.400 & 47310.900 & 47270.600 & 0.000 \\
\hline GaJ76-9 & 35010.467 & 1231.174 & 0.035 & 34393.400 & 33855.500 & 38780.700 & 37349.500 & 1.000 \\
\hline GaJ76-11 & 34904.760 & 567.251 & 0.016 & 35106.700 & 33352.100 & 35162.100 & 37187.100 & 1.000 \\
\hline GaJ76-12 & 35080.993 & 497.483 & 0.014 & 35162.500 & 33343.800 & 35658.200 & 37174.675 & 1.000 \\
\hline GaJ76-10 & 89990.147 & 161.193 & 0.002 & 89907.600 & 89885.700 & 90350.100 & 92565.734 & 1.000 \\
\hline
\end{tabular}

Table 9

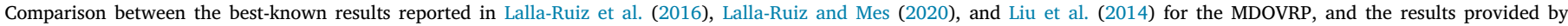
our modified formulation.

\begin{tabular}{|c|c|c|c|c|c|c|c|c|c|}
\hline $\begin{array}{l}\text { Instance } \\
\text { ID }\end{array}$ & $\mathrm{n}$ & $\mathrm{m}$ & $\mathrm{Q}$ & BKR & $\begin{array}{l}\text { Model } \\
\text { solution }\end{array}$ & LB & $\begin{array}{l}\% \text { GAP } \\
\text { from LB }\end{array}$ & $t(s)$ & $\begin{array}{l}\text { Percentage of } \\
\text { improvement }\end{array}$ \\
\hline p01 & 50 & 4 & 80 & $386.18^{a, b, c}$ & $386.18^{*}$ & - & - & 4 & 0.00 \\
\hline p02 & 50 & 4 & 160 & $375.93^{a, b, c}$ & $375.93^{*}$ & - & - & 1 & 0.00 \\
\hline p03 & 75 & 5 & 140 & $474.57^{b, c}$ & $474.57^{*}$ & - & - & 6 & 0.00 \\
\hline p04 & 100 & 2 & 100 & $662.22^{b, c}$ & 662.22 & 648.54 & 2.11 & 7200 & 0.00 \\
\hline p05 & 100 & 2 & 200 & $607.53^{c}$ & $607.53^{*}$ & - & - & 36 & 0.00 \\
\hline p06 & 100 & 3 & 100 & $611.99^{b}$ & $611.99 *$ & - & - & 505 & 0.00 \\
\hline p07 & 100 & 4 & 100 & $608.28^{c}$ & $608.28^{*}$ & - & - & 777 & 0.00 \\
\hline p08 & 249 & 2 & 500 & $2852.56^{b}$ & 2853.57 & 2591.63 & 10.11 & 7200 & -0.04 \\
\hline p09 & 249 & 3 & 500 & $2625.04^{b}$ & 2595.80 & 2431.24 & 6.77 & 7200 & 1.11 \\
\hline p10 & 249 & 4 & 500 & $2528.98^{c}$ & 2488.77 & 2357.88 & 5.55 & 7200 & 1.59 \\
\hline p11 & 249 & 5 & 500 & $2499.25^{c}$ & 2465.28 & 2353.91 & 4.78 & 7200 & 1.36 \\
\hline p12 & 80 & 2 & 60 & $953.26^{a, b, c}$ & $953.26^{*}$ & - & - & 2 & 0.00 \\
\hline p15 & 160 & 4 & 60 & $1885.81^{b, c}$ & $1885.81^{*}$ & - & - & 18 & 0.00 \\
\hline p18 & 240 & 6 & 60 & $2818.36^{b, c}$ & $2818.36^{*}$ & - & - & 65 & 0.00 \\
\hline pr01 & 48 & 4 & 200 & $647.03^{a, b, c}$ & $647.03^{*}$ & - & - & 1 & 0.00 \\
\hline pr02 & 96 & 4 & 195 & $978.82^{b, c}$ & $978.82^{*}$ & - & - & 6 & 0.00 \\
\hline pr03 & 144 & 4 & 190 & $1423.48^{b, c}$ & $1423.48^{*}$ & - & - & 37 & 0.00 \\
\hline pr04 & 192 & 4 & 185 & $1514.07^{c}$ & $1514.07^{*}$ & - & - & 1362 & 0.00 \\
\hline pr05 & 240 & 4 & 180 & $1706.8^{c}$ & 1697.99 & 1639.41 & 3.57 & 7200 & 0.52 \\
\hline pr06 & 288 & 4 & 175 & $1978.46^{c}$ & 1976.47 & 1939.13 & 1.93 & 7200 & 0.10 \\
\hline pr07 & 72 & 6 & 200 & $821.25^{a, b, c}$ & $821.25^{\star}$ & - & - & 1 & 0.00 \\
\hline pr08 & 144 & 6 & 190 & $1254.45^{b, c}$ & $1254.45^{*}$ & - & - & 33 & 0.00 \\
\hline pr09 & 216 & 6 & 180 & $1591.78^{c}$ & $1591.78^{*}$ & - & - & 828 & 0.00 \\
\hline pr10 & 288 & 6 & 170 & $1977.33^{c}$ & 1968.67 & 1921.08 & 2.48 & 7200 & 0.44 \\
\hline
\end{tabular}

*Our formulation reported those solutions as optimal.

use it for comparison as they used approximation methods to solve the problem. Table 9 exhibits the results, where columns 1,2 and 3 denote the instance ID, number of customers and number of depots, respectively. Column 4 displays the best-known result (BKR) reported in Lalla-Ruiz et al. (2016). To make easy to recognize which formulation obtained the best result, a super-index is added to the each value of the fourth column as follows: letter $a$ is used to indicate that it belongs to Liu et al. (2014) while letter $b$ is used for Lalla-Ruiz et al. (2016). In case that both algorithms report the BKR, the two indices are displayed. Column 5 shows the solution obtained by our formulation whereas columns 6 and 7 indicate the best lower bound and the corresponding GAP (in percentage) reported by CPLEX. Finally, columns 8 and 9, display respectively the elapsed CPU time (in seconds) and the percentage of improvement respect to the BKR, calculated as shown in Eq. (17).

Percentage of improvement $=\frac{100 \cdot(B K R-\text { Model solution })}{B K R}$

From Table 9, it can be observed that our formulation outperforms the models previously presented in the literature (Lalla-Ruiz et al., 2016; Lalla-Ruiz \& Mes, 2020). Moreover, our mathematical model 
Table 10

Comparison between the best-known results reported in Brandão (2020) for the MDOVRP and the results obtained by our algorithm.

\begin{tabular}{|c|c|c|c|c|}
\hline $\begin{array}{l}\text { Instance } \\
\text { ID }\end{array}$ & BKS & BSF & $\begin{array}{l}\text { Avg. CPU time } \\
\text { (s) }\end{array}$ & $\begin{array}{l}\text { Percentage of } \\
\text { improvement }\end{array}$ \\
\hline p01 & $386.18^{*}$ & 386.181 & 4.551 & 0.00 \\
\hline p03 & $474.57^{*}$ & 485.196 & 7.628 & -2.24 \\
\hline p04 & $662.22^{a, b}$ & 689.138 & 105.709 & -4.07 \\
\hline p05 & $607.53^{*}$ & 623.033 & 11.950 & -2.55 \\
\hline p08 & $2788.96^{d}$ & 3027.150 & 123.837 & -6.34 \\
\hline p09 & $2578.49^{d}$ & 3049.100 & 134.988 & -18.25 \\
\hline p10 & $2491.44^{d}$ & 2730.140 & 131.284 & -9.58 \\
\hline p11 & $2468.45^{d}$ & 2707.010 & 134.436 & -9.66 \\
\hline p12 & $953.25^{*}$ & 1021.160 & 6.536 & -7.12 \\
\hline p15 & $1885.8^{*}$ & 2044.420 & 39.893 & -8.41 \\
\hline pr04 & $1517.6^{d}$ & 1637.300 & 57.221 & -7.89 \\
\hline pr05 & $1700.19^{d}$ & 1915.100 & 100.717 & -12.64 \\
\hline pr06 & $1985.59^{d}$ & 2152.190 & 173.677 & -8.39 \\
\hline pr07 & $821.25^{\star}$ & 839.350 & 5.398 & -2.20 \\
\hline pr08 & $1254.45^{d}$ & 1325.120 & 27.883 & -5.63 \\
\hline pr09 & $1592.77^{d}$ & 1694.340 & 80.689 & -6.38 \\
\hline pr10 & $1974.56^{d}$ & 2186.160 & 176.598 & -10.72 \\
\hline Average & & & 64.127 & -6.61 \\
\hline
\end{tabular}

*Those solutions are proved as optimal.

solved to optimally $66.67 \%$ ( 16 out of 24 ) of the benchmark instances (the largest one solved with a size of 216 customers and 6 depots), whereas for the remaining, in all but except one, the model improved or tied the BKR. The notes in the BKR column indicate if the value comes from Liu et al. (2014) with an (a), from Lalla-Ruiz et al. (2016) with a (b), or from Lalla-Ruiz and Mes (2020) with a (c). Given that the purpose of this work is not to compare with the state of the art in the MDOVRP, and we are not discussing the performance in terms of computational time. The new best solution for instance $p 10$ is shown in the Appendix, and the new best solutions found for instances in Table 9 are available upon request.

\subsubsection{Algorithm results}

We compare the performance of our metaheuristic procedures with respect to those designed explicitly for the MDOVRP. In particular, we selected the publication of Brandão (2020) since he reported the most recent results in comparison with the relevant algorithms in the literature. As in the model, we modified our algorithm in order to implement it by considering the characteristics of the MDOVRP. Table 10 exhibits the obtained results, where the first column displays the instance ID, columns 2 and 3 show the best known solution (BKS) reported in the literature and the best solution found (BSF) by the algorithm. Column 4 indicates the average CPU time spent over the 15 runs. Finally, the last column presents the GAP of our solution from the BKS. For the calculation of the percentage of improvement, we use Eq. (18):

Percentage of improvement $=\frac{100 \cdot(B K S-B S F)}{B K S}$

In case of the solution is proved optimal, that will be marked with the symbol (*), for the other instances, we consider the value reported by Brandão (2020) as reference, denoting them with the symbol $(d)$.

As it can be seen, the BSF provided by the algorithm reported solutions with an average percentage of improvement of $-6.61 \%$, finding only the BKS for the instances p01 and p02, and going worse up to $-18.76 \%$. Although these results may seem discouraging, our interpretation is that they are caused by the particularity of the design of the metaheuristic to exploit the characteristics of the problem under study. In addition, it can be observed that there is a positive correlation of the number of potential customers and origins with the elapsed CPU time.

When revising the results obtained by our model in detail, we realized that our algorithm has the limitation of creating longer routes, to maximize the usage of the vehicle capacities. Given that the contracting cost in the MDOVRP instances is zero, the optimal solutions reported in the literature sub-utilize the capacity of the vehicles in pursuit of minimizing the total traveled distance. In our problem there is a contracting cost associated with the vehicle capacity, therefore, our algorithm prioritizes to minimize the number of vehicles to use before constructing the routes.

In summary, our algorithm provides acceptable results in comparison with the published methods for simpler problems in the literature. However, for the particular problem under study, the metaheuristic shows an efficient behavior outperforming the results obtained not only by the company's policy but also compared to the mathematical model.

\section{Conclusions}

In this paper, the multi-depot open location routing problem with heterogeneous fixed fleet was introduced, with the aim of seeking to minimize the total transportation costs (fixed and distance-based) by determining which carrier companies and vehicles must be contracted and designing the routes to be assigned to each vehicle. For this problem, a mixed integer formulation and a multi-start metaheuristic algorithm were developed. Both approaches demonstrated effectiveness in terms of the quality of the solution and elapsed computational time.

Regarding the mathematical model, the solver was capable of solving optimally not only the case of study and its variants, but also modified benchmark instances up to 49 nodes with 2 origins and 14 vehicles, in less than two hours. The time spent to solve each of the scenarios derived from the case study was less than $15 \mathrm{~s}$, such that results for a real case can be obtained in "reasonable time". In addition, the proposed formulation was able to deal with the MDOVRP instances, finding new best solutions (some of them proved to be optimal) for the benchmark instances of the well-known MDOVRP, showing its efficiency for dealing with this particular problem.

About the algorithm, it was capable of obtaining competitive results with a significantly shorter computational time for all types of 
instances. Regarding the case of study, the algorithm showed its efficiency in terms of the quality of the solution, the reason for which it can be implemented to solve large-size scenarios if needed. In the case of the MDOVRP benchmark instances, the algorithm experienced acceptable behavior, obtaining results below 5\% away from the BKS for $46 \%$ of the benchmark MDOVRP instances. Regarding the modified benchmark instances to involve heterogeneous fleet, the algorithm achieves high-quality solutions and spent less than $1 \mathrm{~min}$ for the larger ones. Low gaps support this assessment of high quality concerning nonoptimal solutions obtained in the CPU time-limit by the commercial solver.

As can be seen, this research contributes not only by solving a reallife problem of a company but also by introducing a location-routing problem that includes heterogeneous fixed fleet, which could not be found with these particularities in the literature.

Future research lines include to study more realistic scenarios such as considering time windows, split deliveries, or minimizing the total arrival time to the customers instead of the total traveled distance. Additionally, it will be interesting to evaluate the impact of the contracting cost over the total cost and how it determines the configuration of the routes, using a biobjective or bilevel optimization approach.

\section{CRediT authorship contribution statement}

Samuel Nucamendi-Guillén: Conceptualization, Methodology, Software, Validation, Formal analysis, Investigation, Data curation, Writing - original draft, Writing - review \& editing, Visualization, Project administration. Alejandra Gómez Padilla: Conceptualization, Validation, Formal analysis, Investigation, Data curation, Writing - original draft, Writing - review \& editing. Elias OlivaresBenitez: Conceptualization, Validation, Formal analysis, Data curation, Writing - original draft, Writing - review \& editing, Visualization, Supervision, Project administration, Funding acquisition. J. Marcos Moreno-Vega: Conceptualization, Validation, Writing original draft, Writing - review \& editing, Supervision, Funding acquisition.

\section{Declaration of competing interest}

The authors declare that they have no known competing financial interests or personal relationships that could have appeared to influence the work reported in this paper.

\section{Acknowledgments}

This work has been supported by Universidad Panamericana, Mexico [grant number: UP-CI-2020-GDL-07-ING], and by the Agencia Estatal de Investigación (Spain) (project PID2019-104410RB-I00/AEI/ 10.13039/501100011033).

\section{Appendix}

The new best solution for instance P10 is as follows (nodes from 1 to 249 correspond to the customers, whereas nodes $250-253$ refer to the depots):

2501112362312022441992004621663180152

250105132972922720109

250196481281791619318745

250921782104111060158134159

250851691711811627559

25011798240

2501741431410721277915088247

25022216537624316

251223431221468413531181238190

25121457203193101114148269153

251102381311316789248189121106
251112412358222416364125

2512251572496223717769

2515623317613622632782016751

251661684755175172440127147

25124238391021521952

2514219719480113103

25212491301292216190211

252137

25217327195217023058183150

252542041987320716696112213215126116

25218301915133791918572

25212453228652068321736220

25318834

253481186211383323468142

253871547615523223120213912297131205

2531151451002099970108244

2539518235239192184218119

25316071641401727410414125246208

253281561442459415186149

\section{References}

Aksoy, O., \& Kapanoglu, M. (2012). Multi-commodity, multi-depot, heterogenous vehicle pickup and delivery problem for air transportation in the turkish air force. Journal of Aeronautics and Space Technologies, 5(4), 53-57.

Angel-Bello, F., Cardona-Valdés, Y., \& Álvarez, A. (2017). Mixed integer formulations for the multiple minimum latency problem. Operational Research, http://dx.doi.org/ 10.1007/s12351-017-0299-4.

Bezerra, S. N., de Souza, S. R., \& Souza, M. J. F. (2018). A GVNS algorithm for solving the multi-depot vehicle routing problem. Electronic Notes in Discrete Mathematics, 66, 167-174. http://dx.doi.org/10.1016/j.endm.2018.03.022, 5th International Conference on Variable Neighborhood Search.

Braekers, K., Ramaekers, K., \& Van Nieuwenhuyse, I. (2016). The vehicle routing problem: State of the art classification and review. Computers \& Industrial Engineering, 99, 300-313.

Brandão, J. (2020). A memory-based iterated local search algorithm for the multi-depot open vehicle routing problem. European Journal of Operational Research.

Christofides, N., \& Eilon, S. (1969). An algorithm for the vehicle-dispatching problem. Operational Research, 20(3), 309-318.

Chunyu, R., \& Xiaobo, W. (2010). Research on multi-vehicle and multi-depot vehicle routing problem with time windows for electronic commerce. In 2010 international conference on artificial intelligence and computational intelligence, Vol. 1 (pp. 552-555). http://dx.doi.org/10.1109/AICI.2010.121.

Cordeau, J.-F., Gendreau, M., \& Laporte, G. (1997). A tabu search heuristic for periodic and multi-depot vehicle routing problems. Networks: An International Journal, 30(2), 105-119.

Dantzig, G. B., \& Ramser, J. H. (1959). The truck dispatching problem. Management Science, 6(1), 80-91.

Drexl, M., \& Schneider, M. (2015). A survey of variants and extensions of the location-routing problem. European Journal of Operational Research, 241(2), 283308. http://dx.doi.org/10.1016/j.ejor.2014.08.030, URL: http://www.sciencedirect. com/science/article/pii/S0377221714006651.

Eksioglu, B., Vural, A. V., \& Reisman, A. (2009). The vehicle routing problem: A taxonomic review. Computers \& Industrial Engineering, 57(4), 1472-1483.

Euchi, J., \& Chabchoub, H. (2010). A hybrid tabu search to solve the heterogeneous fixed fleet vehicle routing problem. Logistics Research, 2(1), 3-11.

Gillett, B. E., \& Johnson, J. G. (1976). Multi-terminal vehicle-dispatch algorithm. Omega, 4(6), 711-718.

Gillett, B. E., \& Miller, L. R. (1974). A heuristic algorithm for the vehicle-dispatch problem. Operations Research, 22(2), 340-349.

Golden, B., Assad, A., Levy, L., \& Gheysens, F. (1984). The fleet size and mix vehicle routing problem. Computers \& Operations Research, 11(1), 49-66. http://dx.doi.org/ 10.1016/0305-0548(84)90007-8.

Juan, A. A., Pascual, I., Guimarans, D., \& Barrios, B. (2015). Combining biased randomization with iterated local search for solving the multidepot vehicle routing problem. International Transactions in Operational Research, 22(4), 647-667. http: //dx.doi.org/10.1111/itor.12101.

Kim, H., Yang, J., \& Lee, K.-D. (2011). Reverse logistics using a multi-depot VRP approach for recycling end-of-life consumer electronic products in South Korea. International Journal of Sustainable Transportation, 5(5), 289-318.

Koç, Ç., Bektaş, T., Jabali, O., \& Laporte, G. (2016). The impact of depot location, fleet composition and routing on emissions in city logistics. Transportation Research, Part B (Methodological), 84, 81-102. http://dx.doi.org/10.1016/j.trb.2015.12.010, URL: http://www.sciencedirect.com/science/article/pii/S0191261515002714. 
Koulaeian, M., Seidgar, H., Kiani, M., \& Fazlollahtabar, H. (2015). A multi depot simultaneous pickup and delivery problem with balanced allocation of routes to drivers. International Journal of Industrial Engineering: Theory, Applications and Practice, 22(2), 223-242, URL: http://journals.sfu.ca/ijietap/index.php/ijie/article/ view/1379.

Lahyani, R., Coelho, L. C., \& Renaud, J. (2018). Alternative formulations and improved bounds for the multi-depot fleet size and mix vehicle routing problem. OR Spectrum, 40(1), 125-157. http://dx.doi.org/10.1007/s00291-017-0494-y.

Lalla-Ruiz, E., Expósito-Izquierdo, C., Taheripour, S., \& Voß, S. (2016). An improved formulation for the multi-depot open vehicle routing problem. OR Spectrum, 38(1), 175-187.

Lalla-Ruiz, E., \& Mes, M. (2020). Mathematical formulations and improvements for the multi-depot open vehicle routing problem. Optimization Letters, http://dx.doi.org/ 10.1007/s11590-020-01594-z.

Lalla-Ruiz, E., \& Voß, S. (2019). A POPMUSIC approach for the multi-depot cumulative capacitated vehicle routing problem. Optimization Letters, http://dx.doi.org/10. 1007/s11590-018-1376-1.

Liu, R., Jiang, Z., \& Geng, N. (2014). A hybrid genetic algorithm for the multi-depot open vehicle routing problem. OR Spectrum, 36(2), 401-421.

Markov, I., Varone, S., \& Bierlaire, M. (2015). The waste collection VRP with intermediate facilities, a heterogeneous fixed fleet and a flexible assignment of origin and destination depot: Technical Report TRANSP-OR 150212, Transport and Mobility Laboratory, Ecole Polytechnique Fédérale de Lausanne.

Montoya-Torres, J. R., Franco, J. L., Isaza, S. N., Jiménez, H. F., \& Herazo-Padilla, N. (2015). A literature review on the vehicle routing problem with multiple depots. Computers \& Industrial Engineering, 79, 115-129.

Moura, A., \& Oliveira, J. (2005). A GRASP approach to the container-loading problem. IEEE Intelligent Systems, 20, 50-57. http://dx.doi.org/10.1109/MIS.2005.57.

Niu, Y., Yang, Z., Chen, P., \& Xiao, J. (2018). A hybrid tabu search algorithm for a real-world open vehicle routing problem involving fuel consumption constraints. Complexity, 2018, Article 5754908. http://dx.doi.org/10.1155/2018/5754908, 12 pages.

Nucamendi-Guillén, S., Angel-Bello, F., Martínez-Salazar, I., \& Cordero-Franco, A. E. (2018). The cumulative capacitated vehicle routing problem: New formulations and iterated greedy algorithms. Expert Systems with Applications, 113, 315-327. http://dx.doi.org/10.1016/j.eswa.2018.07.025, URL: http://www. sciencedirect.com/science/article/pii/S0957417418304391.

Penna, P. H. V., Subramanian, A., \& Ochi, L. S. (2013). An iterated local search heuristic for the heterogeneous fleet vehicle routing problem. Journal of Heuristics, 19(2), 201-232. http://dx.doi.org/10.1007/s10732-011-9186-y.

Pichka, K., Bajgiran, A. H., Petering, M. E. H., Jang, J., \& Yue, X. (2018). The two echelon open location routing problem: Mathematical model and hybrid heuristic. Computers \& Industrial Engineering, 121, 97-112. http://dx.doi.org/10.1016/j.cie. 2018.05.010.

Salhi, S., Imran, A., \& Wassan, N. A. (2014). The multi-depot vehicle routing problem with heterogeneous vehicle fleet: Formulation and a variable neighborhood search implementation. Computers \& Operations Research, 52, 315-325. http://dx. doi.org/10.1016/j.cor.2013.05.011, URL: http://www.sciencedirect.com/science/ article/pii/S0305054813001408. Recent advances in Variable neighborhood search.
Sariklis, D., \& Powell, S. (2000). A heuristic method for the open vehicle routing problem. The Journal of the Operational Research Society, 51(5), 564-573. http: //dx.doi.org/10.1057/palgrave.jors.2600924.

Schrage, L. (1981). Formulation and structure of more complex/realistic routing and scheduling problems. Networks, 11(2), 229-232. http://dx.doi.org/10.1002/net. 3230110212.

Soto, M., Sevaux, M., Rossi, A., \& Reinholz, A. (2017). Multiple neighborhood search, tabu search and ejection chains for the multi-depot open vehicle routing problem. Computers \& Industrial Engineering, 107, 211-222.

Taillard, E. D. (1999). A heuristic column generation method for the heterogeneous fleet VRP. RAIRO-Operational Research, 33(1), 1-14. http://dx.doi.org/10.1051/ro: 1999101.

Tang, K., Yin, J., \& Man, K. (2010). A genetic-based optimization for multi-depot vehicle routing problems. In Industrial electronics (ISIE), 2010 IEEE international symposium on (pp. 1545-1549). IEEE.

Tarantilis, C., \& Kiranoudis, C. (2002). Distribution of fresh meat. Journal of Food Engineering, 51(1), 85-91.

Ting, C.-J., \& Chen, C.-H. (2013). A multiple ant colony optimization algorithm for the capacitated location routing problem. International Journal of Production Economics, 141(1), 34-44. http://dx.doi.org/10.1016/j.ijpe.2012.06.011, URL: http: //www.sciencedirect.com/science/article/pii/S0925527312002393. Meta-heuristics for manufacturing scheduling and logistics problems.

Verdegay, J. L., Yager, R. R., \& Bonissone, P. P. (2008). On heuristics as a fundamental constituent of soft computing. Fuzzy Sets and Systems, 159, 846-855. http://dx.doi. org/10.1016/j.fss.2007.08.014.

Wang, Q., Ji, Q., \& Chiu, C.-H. (2014). Optimal routing for heterogeneous fixed fleets of multicompartment vehicles. Mathematical Problems in Engineering, 2014, Article 847630. http://dx.doi.org/10.1155/2014/847630, 11 pages.

Wang, G., \& Lin, Z. (2017). Hybrid mosquito host-seeking algorithm for multi-depot vehicle routing problem. In 9th international conference on measuring technology and mechatronics automation (ICMTMA), 2017 (pp. 182-186). IEEE.

Wang, T. J., \& Wu, K. J. (2015). Optimization algorithm for multi-vehicle and multidepot emergency vehicle dispatch problem. Advances in Transportation Studies, 2, 23-30. http://dx.doi.org/10.4399/978885488963703.

Wren, A., \& Holliday, A. (1972). Computer scheduling of vehicles from one or more depots to a number of delivery points. Operational Research Quarterly, 23(3), 333-344.

Yu, V. F., \& Lin, S.-Y. (2015). A simulated annealing heuristic for the open locationrouting problem. Computers \& Operations Research, 62, 184-196. http://dx.doi.org/ 10.1016/j.cor.2014.10.009.

Yu, V. F., Redi, A. A. N. P., Halim, C., \& Jewpanya, P. (2020). The path cover problem: Formulation and a hybrid metaheuristic. 146, 113107. URL: http://www.sciencedirect.com/science/article/pii/S0957417419308243. http://dx. doi.org/10.1016/j.eswa.2019.113107.

Zhao, F., Zhang, L., Zhang, Y., Ma, W., Zhang, C., \& Song, H. (2020). A hybrid discrete water wave optimization algorithm for the no-idle flowshop scheduling problem with total tardiness criterion. Expert Systems with Applications, 146, http: //dx.doi.org/10.1016/j.eswa.2019.113166.

Zhu, X., Yan, R., \& Zhang, Q. (2015). A promoted hybrid heuristic algorithm for two-dimensional multi-depots vehicle routing problem. International Journal of Simulation Modelling, 14(3), 499-510. 